\title{
Influence of Lab Adapted Natural Diet and Microbiota on Life History and Metabolic Phenotype of Drosophila melanogaster
}

\author{
Andrei Bombin *(1), Owen Cunneely, Kira Eickman, Sergei Bombin, Abigail Ruesy, Mengting Su, \\ Abigail Myers, Rachael Cowan and Laura Reed * \\ Department of Biological Sciences, University of Alabama, Tuscaloosa, AL 35487, USA; \\ opcunneely@crimson.ua.edu (O.C.); kmeickman@crimson.ua.edu (K.E.); sbombin@crimson.ua.edu (S.B.); \\ amruesy@crimson.ua.edu (A.R.); msu1@crimson.ua.edu (M.S.); amyers24@crimson.ua.edu (A.M.); \\ racowan@crimson.ua.edu (R.C.) \\ * Correspondence: abombin@crimson.ua.edu (A.B.); lreed1@ua.edu (L.R.)
}

Received: 9 November 2020; Accepted: 9 December 2020; Published: 11 December 2020

\begin{abstract}
Symbiotic microbiota can help its host to overcome nutritional challenges, which is consistent with a holobiont theory of evolution. Our project investigated the effects produced by the microbiota community, acquired from the environment and horizontal transfer, on metabolic traits related to obesity. The study applied a novel approach of raising Drosophila melanogaster, from ten wild-derived genetic lines on naturally fermented peaches, preserving genuine microbial conditions. Larvae raised on the natural and standard lab diets were significantly different in every tested phenotype. Frozen peach food provided nutritional conditions similar to the natural ones and preserved key microbial taxa necessary for survival and development. On the peach diet, the presence of parental microbiota increased the weight and development rate. Larvae raised on each tested diet formed microbial communities distinct from each other. The effect that individual microbial taxa produced on the host varied significantly with changing environmental and genetic conditions, occasionally to the degree of opposite correlations.
\end{abstract}

Keywords: microbiota; 16S; metabolic phenotype; host microbiota interaction

\section{Introduction}

The holobiont theory states that a host and its commensal microbiota possess a metagenome that expresses a synergistic phenotype, which is subjected to evolutionary forces as one complex organism [1]. A phenotype of this unit could be varied by genome modifications of the host, and its commensal bacteria. Metagenomic changes induced by bacteria have more potential for genetic variability and could arise by altering dominant species of bacteria, and acquisition of new strains of microorganisms from an environment [2].

Gut microbiota is one of the most important factors shaping metabolic phenotype and, as a consequence, is a key element in the development of metabolic and autoimmune diseases, cancer, and asthma [3,4]. Alterations in gut microbiota biodiversity and community structure are correlated with the development of the obese phenotype [5,6]. Transfer of the microbiota from an obese to a lean, axenic (microbiota free) individual significantly increases weight gain and adiposity, compared to axenic mice colonized with microbiota community from a lean organism $[5,7,8]$. These results suggest that obesity can be transferred from one individual to another; therefore, exhibiting some characteristics of an infectious disease [8].

Many species of Drosophila use rotting fruit as their primary host, including Drosophila melanogaster, and primarily consume the microorganisms growing in the rot. During colonization of a rotting fruit, 
Drosophila inoculates the substrate with their microbiota [9]. In addition, when females deposit embryos on a substrate, parental microbiota resides on the chorion of the egg [10]. The initial microbiota is gained by the first instar larvae through consumption of the chorion [10]. Later, during feeding, larvae acquire additional microbiota from the environment, representatives of which remain in the larval and pupal intestine until eclosion of the adult fly [11]. Wong et al. [12] showed that parental microbiota transferred with the chorion of the egg could modify the microbial community composition in a food substrate and in the offspring. In addition, the transfer of axenic Drosophila on food substrate would change the food microbial community to resemble the symbiotic microbiota composition that would develop in the host [12].

Symbiotic microbiota play an important role in D. melanogaster development and metabolic phenotype. Axenic flies have a longer development time, lower weight, protein, and glycogen content but higher free glucose and triglyceride levels [13-15]. There are similar negative phenotypic effects on axenic $D$. melanogaster and on those being raised on harmful diets such as high sugar and high fat [13,15-18]. Although axenic Drosophila consume less food, their energy storage indices (triglyceride, glucose, glycogen, and trehalose levels) stay significantly higher than that of conventional flies [19]. In addition, the presence of the commensal microbiota allows Drosophila to maximize their lifespan and reproductive output [4]. Dobson et al. [14] showed that the abundance of specific bacterial taxa is associated with a change in metabolic phenotype: Acetobacter, Gluconobacter and Komagataeibacter are negatively correlated with a fly's energy storage index. Lactobacillus is positively associated with triglyceride concentration and Achromobacter and Xanthomonadaceae have a positive correlation with glycogen levels $[20,21]$. In addition, Drosophila microbiota varies across genetic backgrounds, which makes it possible to establish associations between a host's genes and microbiota dependent metabolic responses and particular symbiotic species [14,21,22].

Symbiotic microbiota allow Drosophila to overcome the nutritional limitation of their diet. Shin et al. [23] showed that axenic larvae raised on a casamino acid diet experienced a $90 \%$ body size reduction and were not able to survive to form pupae. However, the presence of only one bacterial species, Acetobacter pomorum, could restore survival and the normal rate of larval development via induction of the hosts' insulin-like growth factor signaling [23]. Leitão-Gonçalves et al. [4] demonstrated that axenic flies express a strong preference for yeast-rich food due to their demand for essential amino acids. A. promorum and several Lactobacilli species are able to suppress the yeast appetite in Drosophila and shift the flies' nutritional preference toward high-sugar concentration diets [4]. Their change in nutritional preference may be explained by competition between the host and its symbiotic microbiota for available sugars and through production of essential amino acids by the microbial community [4].

Microbiota composition of lab dwelling Drosophila primarily consists of Acetobacteraceae, Enterococcaceae, and Lactobacillaceae families [22,24]. Within these families, the influence of Acetobacter tropicalis, Enterococcus faecalis, Lactobacillus brevis, and Lactobacisul plantarum on a host's metabolic phenotype has been studied more than others $[15,22,23]$. However, the microbiota of wild fly populations may differ in the diversity and the abundance of dominant species [24]. In the lab, Drosophila raised on fruits still exhibit a more complex and diverse community of symbiotic microbes compared to conventionally raised flies [25]. Lab food preservatives, especially methylparaben sodium salt (moldex), largely contribute to the difference between natural and laboratory associated microbiota communities [26]. Therefore, studying the evolutionary relationship of Drosophila and its microbiota, and the symbiont's influence on fly's metabolic phenotype only on standard lab microbiota, may be insufficient to understand the natural relationship and coevolution of the fly and its microbiota.

With this work we wanted to address a series of specific questions and hypotheses:

Hypothesis 1. How does a natural diet with a naturally occurring and/or maternally inherited community of microbes influence the life history and metabolic phenotypes of flies relative to a standard lab diet? Is there genetic variation in the phenotypic response to nutritional change and dietary and parental microbiota availability? We hypothesized that larvae raised on a natural diet will exhibit different life history traits and metabolic 
phenotypes, comparing with larvae raised on a standard lab diet. We hypothesized that the presence of a maternally transmitted microbiota will significantly impact larvae phenotypes, and that this impact may vary across dietary treatments. Given prior findings on the roles of genetic variation on metabolic phenotypes, we hypothesized that there is genetic variation in phenotype that interacts with the dietary conditions and the availability of maternally transmitted microbiota.

Hypothesis 2. Will the symbiotic microbiota community of the larvae raised on the natural diet be different from the lab food raised larvae? Will the presence of maternally inherited microbiota influence the formation of microbial communities? Is the microbiota community variable across host genotypes? We hypothesized that the gut microbial community composition and diversity will vary substantially across both dietary and parental microbiota conditions. We hypothesized that the maternally transmitted microbiota will have "founder effects" in the formation of the larval gut microbiome. We hypothesized that the composition of the microbial community will exhibit variation with host genotype.

Hypothesis 3. Will specific microbial taxa and/or microbiota communities as a whole influence the larvae phenotypes differently across diets and genotypes? We hypothesized that some microbial taxa will have consistent correlations with host phenotype across diets and treatments while others will have a diet or treatment specific relationship.

\section{Materials and Methods}

\subsection{Food Preparation}

Under hot and humid conditions on 28 August 2017, we put approximately 200 peaches outdoors at $33.2598614^{\circ}$ latitude and $-87.4919516^{\circ}$ longitude (Tuscaloosa area, Alabama) and allowed them to decay for six days. Peaches were selected as fruits that are locally grown in Alabama and therefore might provide nutritional conditions experienced by local Drosophila groups. The six day period was chosen as our preliminary study indicated that this period of time produced a nutritional media that supported the development of more flies from first instar to adult stages than two, four, eight, or twelve-day periods. On 3 September 2017, the fruits were collected, manually ground, and stored in freezers at $-20^{\circ} \mathrm{C}$. Peach food (PR) preparation protocol was the following: we allowed approximately one liter of the peach food to thaw, homogenized it with an immersion blender, and distributed it into vials, with approximately $10 \mathrm{~mL}$ of food per $47 \mathrm{~mL}$ "narrow" Drosophila vial. In order to prepare autoclaved peach food (PA), vials containing peach food were autoclaved for $25 \mathrm{~min}$ at $121^{\circ} \mathrm{C}$. Regular Drosophila lab food (R) was cooked according to the protocol described in previous works. Briefly, it was composed of cornmeal $(60 \mathrm{~g})$, yeast $(12 \mathrm{~g})$, agar $(6 \mathrm{~g})$, water $(1 \mathrm{~L})$, tegosept $(13 \mathrm{~mL})$, propionic acid $(6 \mathrm{~mL})$, and molasses $(51 \mathrm{~mL})$. [18,27].

To ensure that the autoclaved peach food did not contain any live microorganisms, we used $1 \mathrm{~g}$ of autoclaved and non-autoclaved peach materials and diluted them in $9 \mathrm{~mL}$ of sterile phosphate-buffered saline solution (PBS) [4,28]. Then, we performed a serial dilution in PBS to get food dilutions [29]. We mixed $1 \mathrm{~mL}$ of each dilution with standard methods agar (criterion) via the pour plate method [29]. The agar was prepared according to the manufacturer's directions. Samples were incubated at $35^{\circ} \mathrm{C}$ for $48 \mathrm{~h}$ and visually evaluated for the presence of microbial colonies [30]. The independent variable for the diet component will be referred to as $\mathrm{D}$.

\subsection{Drosophila Stocks and Husbandry}

We used 10 naturally derived genetic lines created by the DGRP2 project: 142, 153, 440, 748, $787,801,802,805,861$, and 882 [31,32]. These genotypes were chosen as they showed a large range of reaction norms of pupae weight across diets in the previous studies of the Reed's lab and our preliminary study. We chose to focus only on Wolbachia positive stocks to avoid the confounding factors of phenotypic variation due to Wolbachia infection status, instead aiming to evaluate the influence of 
broad bacterial community on larvae phenotype. Stocks (on a molasses-based lab diet as described above) and experimental flies were maintained at constant temperature of $25{ }^{\circ} \mathrm{C}, 50 \%$ humidity, and 12-h light/dark cycle $[16,18,27]$. The independent variable for the genetic component will be referred to as $\mathrm{G}$.

\subsection{Drosophila Embryos Sterilization}

In order to remove parental microbiota, we sterilized 12-h old embryos with subsequent two-minute washes in $2.5 \%$ active hypochlorite solution, $70 \%$ ethanol solution, and sterilized distilled water $[4,28]$. After sterilization, embryos were placed on the apple agar plates, and incubated until the first instar stage under fly-rearing conditions described above [33]. The non-sterilized control embryos (NS) were allowed to develop for $24 \mathrm{~h}$ (until the 1st instar larvae stage) on the apple agar plates, on which they had been deposited. In order to demonstrate that sterilized embryos did not possess parental microbiota, 20 sterilized 1st instar larvae were collected and grinded in $200 \mu \mathrm{L}$ of the sterile PBS using a mechanical homogenizer. The resulting mixtures were plated on nutrient and standard method agars (criterion). The agars were prepared according to the manufacturer's directions. Plates were incubated for $48 \mathrm{~h}$ at $35^{\circ} \mathrm{C}$ and visually evaluated for the presence of microbial colonies [30]. NS larvae were used as the positive control according to the same procedure. All flies' stocks were maintained in the lab for more than 100 generations on the cornmeal-molasses diet in a common incubator with controlled humidity and photoperiod. Therefore, any difference in parental microbiota composition observed between genetic lines at the initiation of the experiment are likely to be attributed to the genetic and not environmental factors. The independent variable for the sterilization treatment component will be referred to as $\mathrm{T}$.

\subsection{Larvae Rearing and Collection}

In three separate time periods (30 days apart), 50 sterilized and non-sterilized larvae, of each genetic line were put in at least three vials of PA, PR, and R food, each. The independent variable for the time period (round) component will be referred to as Ro. Larvae were allowed to develop until the late third instar wandering stage (when they stopped moving but before the pupation started) and then collected in micro centrifuge tubes with sterile Ringer's solution and immediately stored at $-20^{\circ} \mathrm{C}$ [33]. Each vial was checked for the presence of larvae, at the right developmental stage, four times per day at $9 \mathrm{am}, 11 \mathrm{am}, 2: 30 \mathrm{pm}$, and $5 \mathrm{pm}$ for 18 days after larval colonization. Prior to metabolic assays, larvae were inspected for the presence of any damage (damaged ones were removed), and cleaned with at least two washes in a sterile Ringer's solution. Since we tried to minimize the experimental error potentially introduced by variation in time between individual collections of larvae and between genetic lines, larvae were frozen immediately after collections (larvae of the same diet/genetic/line/treatment/round were pulled together across the vials). We were not able to determine the sex of the frozen larvae and therefore did not account for it in our assays. Thus, there may have been sex-specific responses to these experimental conditions we were unable to account for in this study.

\subsection{Measuring Experimental Phenotypes}

Survival: The total number of third instar larvae collected per vial was divided by the number of first instar larvae used to seed the vial (50) to evaluate the proportion of larvae that survived till the late 3rd instar stage (an average of 11 samples per unique diet/treatment/genetic line combination). Developmental rate: The developmental rate, in days, was calculated for each larva individually, from the day it was put in the food vial to the collection date. We then calculated median developmental time per vial and used it in our statistical analysis (on the average nine samples per unique diet/treatment/genetic line combination) [11]. Weight: In order to measure the dry weight, larvae were taken from the $-20{ }^{\circ} \mathrm{C}$ freezer, allowed to reach room temperature and placed in a VWR standard oven at $37^{\circ} \mathrm{C}$ overnight. After drying, each larva was weighed individually using Mettler Toledo XS 105 microbalance. Weights were recorded with LabX direct software v. 2.2 (on the 
average 27 samples per unique diet/treatment/genetic line combination). Triglyceride: With the exception of four samples (due to low survival of larvae of certain diet/treatment/genetic line/round combinations), we homogenized 10 larvae per sample to determine total triglyceride concentration using the Sigma Triglyceride Determination Kit [18,34-36]. Results were adjusted to represent the average triglyceride concentration per milligram of dry larval weight (on the average six samples per unique diet/treatment/genetic line combination). Protein: Protein concentrations were quantified using the Bradford's method with 10 homogenized larvae per sample (with the exception of $3 \%$ of the samples in which we used one to nine larvae, due to especially low survival rates of the specific groups) $[18,37]$. Protein concentrations were averaged to represent the protein concentration per milligram of dry larvae weight (on the average 12 samples per unique diet/treatment/genetic line combination). Glucose: For most of the samples, combined trehalose and glucose concentrations were quantified via homogenization of 10 larvae (with the exception of $5 \%$ of the samples in which we used four to nine larvae) with subsequent overnight incubation in $1 \mu \mathrm{g} / \mathrm{mL}$ trehalase solution and further application of the Sigma Glucose Determination Kit $[16,18,38]$. Glucose concentrations were averaged and adjusted to represent the amount of glucose per milligram of dry weight (on the average six samples per unique diet/treatment/genetic line combination).

To assess the triglyceride, protein and glucose concentrations in the diets, we used freshly unfrozen food and unfrozen food that was incubated in the food vials for seven days at $25^{\circ} \mathrm{C}$, in the same incubator as the experimental fly stocks, where larvae development was taking place. For the food assays, we used the same procedure as for the larvae but with $12.35 \mathrm{mg}$ of the food sample. For the glucose assay, instead of incubating samples in trehalase, we incubated them in $100 \mu \mathrm{L}$ of $1 \mathrm{mg} / \mu \mathrm{L}$ solution of invertase (to convert sucrose into glucose; Ward's Natural Science) overnight at $37^{\circ} \mathrm{C}$. For analysis, the results were adjusted to represent the amount of the measured compound per milligram of the sample. These results can be viewed in S1.2-S1.3.

\subsection{DNA Extraction and Sequencing}

DNA was extracted from 10 larvae with the Qiagen blood and tissue DNA extraction kit according to the standard protocol, with overnight incubation of the samples in proteinase $\mathrm{K}$ at $56^{\circ} \mathrm{C}$. Prior to sequencing, the quality and purity of DNA extractions were confirmed with PCR, including negative controls, with the same primers used for the sequencing protocol and gel electrophoresis. DNA extractions were used for sequencing the V4 region of the $16 \mathrm{~S}$ ribosomal RNA subunit (16S rRNA), which was performed in the Microbiome Core Facility of The University of Alabama in Birmingham, AL according to the previously published method on the Illumina MiSeq platform [39].

Trimmomatic-0.36 [40] was used to process demultiplexed DNA sequences. Standard Illumina-specific barcode sequences, all sequences with less than 36 bases, and leading and trailing low-quality bases were all removed using the default settings of the Trimmomatic-0.36 program. The USEARCH-fastq_mergepairs tool was used to combine forward and reverse readings. All reads with an expected error greater than 1 were removed, and chimeric reads and singletons. Combined readings without a merging pair were filtered using fastq_filter command. The -unoise 3 tool was used to cluster readings into zero-radius OTU (ZOTU) 100\% identity. OTUs were then designated with the lowest taxonomic rank using the UCULT algorithm implemented in QUIME 1.9.1 [41,42] along with SILVA reference database version 132 [43]. Using SILVA v. 132 database, PyNAST [44] with default options was used for sequence alignment. The phylogenetic trees of ZOTUs and OTUs were assembled using the default options of QUIIME 1.9.1 with the FastTree program [45]. Alpha and beta diversities were rarefied with QUIIME 1.91 -single_rarefaction.py using the-subsample_multinomial option in order to subsample the replacements. Rarefaction for all samples was performed to the depth of 4500 reads. This was the lowest possible number of reads between samples. 


\subsection{Statistical Analysis}

Data transformation. Normality tests, data transformations, and statistical models were done with JMP Pro 14.0. Phenotype measurements were tested for normality with the Shapiro-Wilk test and an outlier box plot. Only larvae survival showed normal distribution. Therefore, all other phenotypic measurements data were transformed. We performed a cube root transformation on the data for development rate and glucose by weight, a square root transformation on data for weight and protein by weight, and a log transformation on data for triglyceride by weight concentrations. The bacterial abundance was $\log (x+1)$ transformed for all parametric analyses.

Bacterial Diversity. Alpha and beta diversities were computed in QIIME v. 1.9.1. To estimate alpha diversity, we used Shannon, Simpson, and PD Whole Tree metrices. As all of the alpha diversity indices were not normally distributed, we performed a pairwise comparison of them with a Wilcoxon rank-sum test in R v. 3.5.1 with "matrixTests" package v. 0.17 [46]. Beta diversity was estimated with Bray-Curtis and weighted Unifrac distances. The similarity between each sample's beta diversity distance was evaluated via hierarchical clustering, applying a ward method for distance calculation, and visualized with a constellation plot in JMP v. 14.0.

Statistical modeling: In order to evaluate the contribution of each variable and their interactive effect to each phenotypic development, we used standard least squares model with model effects to include diet $(D)$, genotype $(G)$, sterilization treatment $(T)$, and their specific interactive effect: $\mathrm{D} \times \mathrm{G}$ (diet-by-genotype), $\mathrm{D} \times \mathrm{T}$ (diet-by-treatment), $\mathrm{G} \times \mathrm{T}$ (genotype-by-treatment), and $\mathrm{D} \times \mathrm{G} \times \mathrm{T}$ (diet-by-genotype-by-treatment). In order to verify that the built model fits the data, a lack of fit test was performed. If the time period of the experiment (Ro) and/or the variance between the colorimetric assay runs (triglyceride, protein, and glucose) $(\mathrm{P})$ produced a significant effect, these variables were included in the model's effects, unless their addition caused the model to fail the lack of fit test. Thus, the models for larvae survival, development time, and weight were the following:

$$
\begin{aligned}
y_{i}=\beta_{0}+\beta_{1} D_{1 i}+\beta_{2} G_{2 i} & +\beta_{3} T_{3 i}+\beta_{4}(D * G)_{4 i}+\beta_{5}(D * T)_{5 i}+\beta_{6}(G * T)_{6 i} \\
& +\beta_{7}(D * G * T)_{i 7}+\varepsilon_{i}
\end{aligned}
$$

For triglyceride by weight:

$$
\begin{gathered}
y_{i}=\beta_{0}+\beta_{1} D_{1 i}+\beta_{2} G_{2 i}+\beta_{3} T_{3 i}+\beta_{4}(D * G)_{4 i}+\beta_{5}(D * T)_{5 i}+\beta_{6}(G * T)_{6 i} \\
+\beta_{7}(D * G * T)_{i 7}+\beta_{8} P_{8 i}+\varepsilon_{i}
\end{gathered}
$$

Additionally, for protein and glucose by weight:

$$
\begin{aligned}
y_{i}=\beta_{0}+\beta_{1} D_{1 i} & +\beta_{2} G_{2 i}+\beta_{3} T_{3 i}+\beta_{4}(D * G)_{4 i}+\beta_{5}(D * T)_{5 i}+\beta_{6}(G * T)_{6 i} \\
& +\beta_{7}(D * G * T)_{i 7}+\beta_{8} P_{8 i}+\beta_{9} R_{9 i}+\varepsilon_{i}
\end{aligned}
$$

where $y_{i}$ is the response, $\beta$ values are constants, and $\varepsilon_{i}$ is a random error term.

All models for interactive effects of diet, genotype, and treatment were done with all 10 genetic lines, with the exception of glucose, which was done without 861 due to the low survival rate of this genetic line.

In order to verify that the built model fits the data, a lack of fit test was performed. Only the models with non-significant lack of fit $p$-value were kept. To assess the pairwise difference between diets (R vs. PR and PR vs. PA) and treatments (NS vs. S), we used the least square model with one main explanatory variable of interest (diet or treatment). We also included time period and assay plate variance as additional explanatory variables if they produced a significant effect. The pairwise comparisons were performed with a post-hoc Student's $t$-test.

Bacterial abundance: In order to evaluate if the diet and treatment could serve as categorical predictors for classification of the larvae bacterial samples, we performed discriminant analysis at phylum, class, order, family, and genus taxonomic levels, and at the level of individual ZOTUs. 
The results were visualized with a canonical plot in JMP v. 14.0 (JMP manual). For the 10 most abundant representatives of each taxonomic level, we applied the linear covariance method for the discriminant analysis, which allowed us to visualize the covariates in the form of vector rays. Using this method allowed us to represent which of them drove the separation of the clusters (JMP manual). The length of the ray is correlated with the strength of the impact that it produced on the samples to be separated in the vector direction, on a canonical plot. When we ran the analysis with all identified taxa, we applied a wide linear method for the discriminant analysis. To compare the abundance of bacterial taxa between the diets and treatments, we performed a Wilcoxon test as described above. The $p$ values were adjusted for the false discovery rate with Benjamini-Hochberg correction and added to data tables as FDR $p$ (False discovery rate adjusted $p$ value). The threshold for the value of FDR $p$ that should be considered significant could be subjective and vary from 0.25 to 0.05 among microbiology studies $[47,48]$. To evaluate the interactive effect of the variables on the abundance of each identified bacterial taxa, we used the three-way linear interaction model.

$$
\begin{gathered}
y_{i}=\beta_{0}+\beta_{1} D_{1 i}+\beta_{2} G_{2 i}+\beta_{3} T_{3 i}+\beta_{4}(D * G)_{4 i}+\beta_{5}(D * T)_{5 i}+\beta_{6}(G * T)_{6 i} \\
+\beta_{7}(D * G * T)_{i 7}+\beta_{8} R_{8 i}+\varepsilon_{i}
\end{gathered}
$$

In order to identify the correlations between larvae phenotypes and bacterial abundances, we found the mean phenotype for each combination of diet, genetic line, treatment, and round. Spearman's rank correlation between the microbial abundances and tested phenotypes was calculated with Hmisc v. 4.3-0 in R v. 3.5.1, with the adjustment of $p$ values for FDR $p$ as described above. We also tested the possible interactive effect of each identified bacterial taxa and one of the independent variables on the formation of the tested phenotypes according to the formula:

$$
y_{i}=\beta_{0}+\beta_{1} x_{1 i}+\beta_{2} x_{2 i}+\beta_{3}\left(x_{1 i} * x_{2 i}\right)_{3 i}+\beta_{4} R_{4 i}+\varepsilon_{i}
$$

where, $x_{1}$ was the abundance of the microbial taxa, $x_{2}$ was one of the independent variables $(D, G$, or $\mathrm{T}$ ), and $\mathrm{R}$ was the time component. Development, weight, triglyceride, protein, and glucose were normalized with $\log$, square root, $\log$, $\log$, and cube root, transformations respectively.

\section{Results}

3.1. There Are Substantial Differences in the Life History and Metabolic Phenotypes for Larvae Raised on a Natural Peach Diet with a Naturally Occurring and/or Maternally Inherited Community of Bacteria Relative to a Standard Lab Diet

3.1.1. Larvae Raised on a Natural Diet Exhibited Different Life History Traits and Metabolic Phenotypes from Larvae Raised on a Standard Lab Diet

Survival: The proportion of larvae that survived on the lab diet was significantly higher than the larval survival on a natural peach diet regardless of sterilization (Figure 1A, Table 1 and Supplementary Table S1.1). The undisturbed bacterial community of the peach diet produced a significant positive effect on larvae survival, when compared with the autoclaved peach diet (Figure 1A, Table 1 and Supplementary Table S1.1). Development rate: Within both controlled and sterilized treatments, larvae developed faster on the regular lab diet compared to the natural diet and faster on the regular peach food compared to the autoclaved peach food (Figure 1B, Table 1 and Supplementary Table S1.1). Weight: The third instar larvae raised on the lab food were significantly heavier than those that were raised on the natural diet (Figure 1C, Table 1 and Supplementary Table S1.1). Among the peach diets, larvae consuming the autoclaved diet were significantly lighter (Figure 1C, Table 1 and Supplementary Table S1.1), especially for larvae deprived of parental microbiota, suggesting that under natural nutritional conditions, microorganisms may facilitate growth and weight gain of the larvae. Triglyceride: Although fresh and incubated $\mathrm{R}$ food had higher triglyceride concentrations than the PR food (Supplementary Table S1.2 and Supplementary Table S1.3), larvae raised on the PR 
diet had significantly higher triglyceride concentrations by weight than those that were raised on a lab diet, independent of sterilization treatment (Figure 1D, Table 1 and Supplementary Table S1.1). Incubated autoclaved peach food had significantly higher triglyceride content compared with regular peach food (Supplementary Table S1.3) and produced larvae with higher triglyceride concentration by weight (Figure 1D, Table 1 and Supplementary Table S1.1). Protein: Independent of the treatment, larvae raised on the regular lab food had higher protein by weight concentrations compared to larvae raised on peach food (Figure 1E, Table 1 and Supplementary Table S1.1). Larvae raised on the PA diet had significantly higher protein by weight levels compared to PR raised larvae, but only in the absence of the parental bacterial community (Figure 1E, Table 1 and Supplementary Table S1.1). Evaluating the protein quantity in fresh food itself, we found significantly higher protein concentration in the regular lab diet compared to the peach diet (Supplementary Table S1.2). Between fresh peach diets there was no significant difference in protein (Supplementary Table S1.2). However, after incubation, autoclaved peach food had significantly less protein than regular peach food (Supplementary Table S1.3). Glucose: Larvae raised on a lab food diet had significantly higher glucose concentrations than larvae raised on the peach food diet (Figure 1F, Table 1 and Supplementary Table S1.1). Larvae that consumed PR food had lower glucose by weight concentrations compared with larvae raised on the autoclaved version, which was consistent with our hypothesis (Figure 1F, Table 1 and Supplementary Table S1.1). Interestingly, the fresh peach food itself had a significantly higher glucose concentration than the lab food (Supplementary Table S1.2). However, after incubating the peach food, the concentration of glucose was lower in PR than in both R and PA diets (Supplementary Table S1.3), suggesting a strong impact from the live bacterial community.

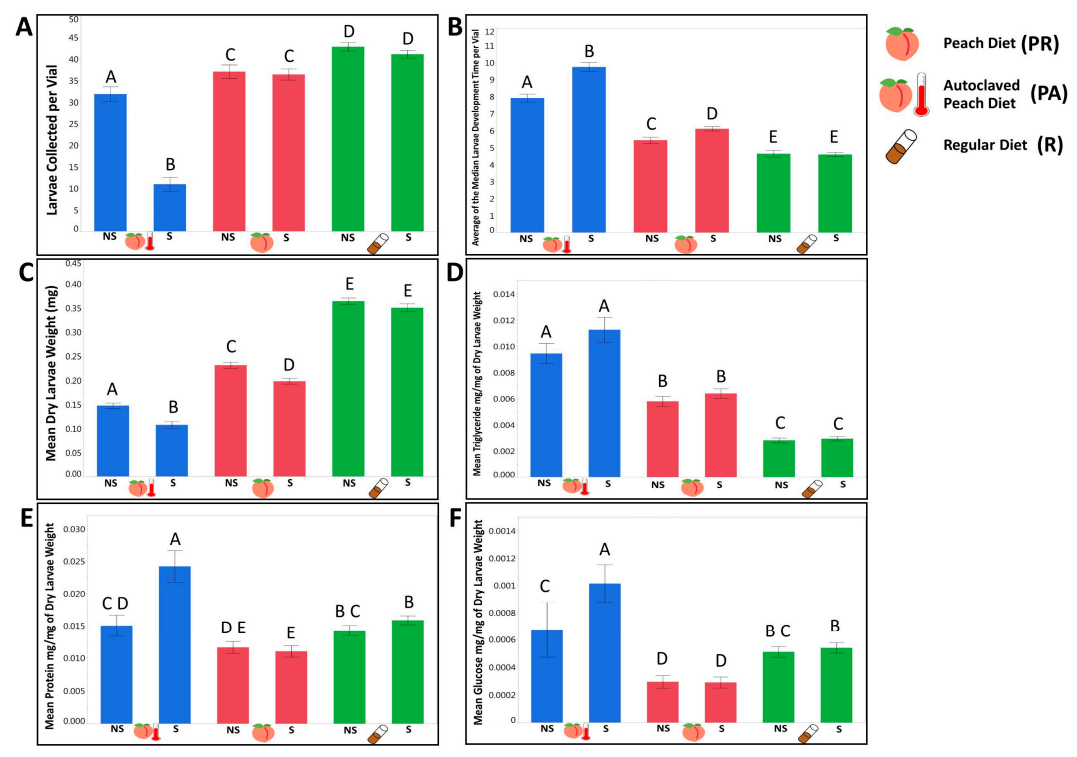

Figure 1. The presence of dietary and/or maternally inherited bacteria substantially impacts metabolic and life history phenotypes in flies. (A) Mean survival until the late 3rd instar stage is increased on lab diet and with the presence of bacteria on peach diets; (B) mean development time is decreased on a lab diet and with the presence of dietary and/or maternal bacteria on peach diets; (C) mean larval weight is higher on a lab diet and in the presence of bacteria on peach diets; (D) triglyceride concentrations by weight are reduced on a lab diet and in the presence of environmental bacteria on peach diets; (E) protein by weight is lower on a peach diet, in the presence of bacteria; and (F) glucose by weight is lowest on a peach diet in the presence of bacteria. Error bars indicated one standard error of the mean. S stands for sterilized larvae and NS stands for non-sterilized larvae. Bars that do not have common letters above them represent significantly different groups $(p<0.05)$ following a pairwise post hoc Student's $t$-test. 
Table 1. The difference in larvae phenotypes based on the diet. Comparison of larvae life history traits and metabolic phenotypes between larvae, from all 10 genetic lines, raised on regular lab diet (R), peach $\operatorname{diet}(\mathrm{PR})$, and autoclaved peach diet (PA). NS stands for non-sterilized larvae, $\mathrm{S}$ stands for sterilized larvae. Asterisks indicate the uncorrected significance of comparisons $p<0.001^{* * *}$ and $p<0.01^{* *}$.

\begin{tabular}{|c|c|c|c|c|c|c|}
\hline Treatment & Survival & Development & Weight & Triglyceride & Protein & Glucose \\
\hline NS & $\mathrm{R}>\mathrm{PR} * *$ & $\mathrm{R}<\mathrm{PR} * * *$ & $\mathrm{R}>\mathrm{PR} * * *$ & $\mathrm{R}<\mathrm{PR} \mathrm{R}^{* * *}$ & $\mathrm{R}>\mathrm{PR} * *$ & $\mathrm{R}>\mathrm{PR} \mathrm{R}^{* * *}$ \\
\hline$S$ & $\mathrm{R}>\mathrm{PR} * *$ & $\mathrm{R}<\mathrm{PR}{ }^{* * *}$ & $\mathrm{R}>\mathrm{PR} * * *$ & $\mathrm{R}<\mathrm{PR} \mathrm{R}^{* * *}$ & $\mathrm{R}>\mathrm{PR} * * *$ & $\mathrm{R}>\mathrm{PR} * * *$ \\
\hline NS & $\mathrm{PR}>\mathrm{PA} * * *$ & $\mathrm{PR}<\mathrm{PA}{ }^{* * *}$ & $\mathrm{PR}>\mathrm{PA}{ }^{* * *}$ & $\mathrm{PR}<\mathrm{PA}^{* * *}$ & $\mathrm{PR}<\mathrm{PA}$ & $\mathrm{PR}<\mathrm{PA} * *$ \\
\hline$S$ & $\mathrm{PR}>\mathrm{PA}^{* * *}$ & $\mathrm{PR}<\mathrm{PA}^{* * *}$ & $\mathrm{PR}>\mathrm{PA}^{* * *}$ & $\mathrm{PR}<\mathrm{PA}^{* * *}$ & $\mathrm{PR}<\mathrm{PA}^{* * *}$ & $\mathrm{PR}<\mathrm{PA}^{* * *}$ \\
\hline
\end{tabular}

3.1.2. We Observed That the Presence of a Maternally Transmitted Bacteria Significantly Impacted Larvae Phenotypes, and That Impact Varied across Dietary Treatments

Survival: The presence of the parental bacteria significantly enhanced overall survival of larvae on the autoclaved peach diet but did not produce a significant effect on larval survival on the lab diet or the non-autoclaved peach diet (Figure 1A, Table 2 and Supplementary Table S1.4). This suggested that the availability of bacterial taxa was necessary for a successful transition of the larvae through the instar stages under natural nutritional conditions, and that these bacterial taxa could be acquired from the food substrate if available and/or inherited maternally. Development rate: Presence of parental bacteria on the peach diet reduced the number of days necessary for larvae to reach the third instar stage on the autoclaved peach diet but not on the regular lab diet (Figure 1B, Table 2 and Supplementary Table S1.4), suggesting that under natural nutritional conditions, maternal microbes might influence the larval developmental rate independent of bacteria acquired from the food substrate. Weight: Maternally inherited bacteria produced a significant positive effect on larval weight on all of the tested diets (Figure 1C, Table 2 and Supplementary Table S1.4). This indicated the universality of their influence on larval growth across food substrates. Triglyceride: Parental bacteria did not influence the triglyceride concentrations significantly on any diet (Figure 1D, Table 2 and Supplementary Table S1.4). Protein. Evaluating the role of parental bacteria, we observed that sterilized larvae had higher protein by weight concentrations but only on the PA diet (Figure 1E, Table 2 and Supplementary Table S1.4). This suggested that the core bacteria involved in a natural metabolic phenotype formation might be inherited or acquired from the environment. Glucose: The parental bacteria reduced the glucose by weight concentrations only on PA food (Figure 1F, Table 2, and Supplementary Table S1.4), indicating that both parental and environmental microbial taxa might be sufficient to reduce glucose concentrations in larvae.

Table 2. The influence of parental microbiota on larvae phenotypes. Comparison of larvae life history traits and metabolic phenotypes between larvae, from all 10 genetic lines, raised on regular lab diet (R), peach diet (PR), and autoclaved peach diet (PA). NS stands for non-sterilized larvae, $\mathrm{S}$ stands for sterilized larvae. Asterisks indicate the uncorrected significance of comparisons $p<0.001^{* * *}$ and $p<0.01^{* *}$.

\begin{tabular}{|c|c|c|c|c|c|c|c|}
\hline Genetic Line & Diet & Survival & Development & Weight & Triglyceride & Protein & Glucose \\
\hline All & PA & $S<N S^{* * *}$ & $S>N S * * *$ & $S<N S * * *$ & $S>N S$ & $\mathrm{~S}>\mathrm{NS} \mathrm{N}^{* *}$ & $\mathrm{~S}>\mathrm{NS}{ }^{* *}$ \\
\hline All & PR & $\mathrm{S}<\mathrm{NS}$ & $\mathrm{S}>\mathrm{NS} * * *$ & $\mathrm{~S}<\mathrm{NS} * * *$ & $\mathrm{~S}>\mathrm{NS}$ & $\mathrm{S}<\mathrm{NS}$ & $\mathrm{S}>\mathrm{NS}$ \\
\hline All & $\mathrm{R}$ & $\mathrm{S}<\mathrm{NS}$ & $\mathrm{S}>\mathrm{NS}$ & $\mathrm{S}<\mathrm{NS}$ & $\mathrm{S}>\mathrm{NS}$ & $\mathrm{S}>\mathrm{NS}$ & $\mathrm{S}>\mathrm{NS}$ \\
\hline
\end{tabular}

3.1.3. Evaluating the Contribution of Tested Independent Variables on Larvae Phenotypes, We Observed a Genetic Variation in Most of the Tested Life History Traits and Phenotypes that Interacted with the Dietary Conditions and the Availability of Maternally Transmitted Microbiota

Survival: All of the independent variables included in the model produced a significant effect on larval survival until the late third instar stage. Of the tested variables, diet was the strongest predictor of survival, followed by the interactive effect of the diet by treatment and genetic line (Table 3 and Supplementary Table S1.5). Development: The development rate of the larvae was 
significantly influenced by diet, genotype, and treatment (Table 3 and Supplementary Table S1.5). Among the specific interaction of these variables, only $\mathrm{D} \times \mathrm{T}$ and $\mathrm{G} \times \mathrm{T}$ produced a significant effect on development (Table 3 and Supplementary Table S1.5). Diet was the key factor that influenced the time necessary for the larvae to reach the late third instar stage and explained almost half of all variance followed by the genetic line (Table 3 and Supplementary Table S1.5). The combination of the rest of the variables was responsible only for $8.42 \%$ of variation in developmental time (Table 3 and Supplementary Table S1.5).

Table 3. The contribution of diet, genotype, treatment, and their interactive effects on formation of larvae life history traits and metabolic phenotypes. VE stands for variance explained, by each independent variable. Asterisks indicate the significance of comparisons $p<0.001^{* * *}, p<0.01^{* *}$, and $p<0.05^{*}$.

\begin{tabular}{|c|c|c|c|c|c|c|}
\hline Independent Variable & Survival & Development & Weight & Triglyceride & Protein & Glucose \\
\hline Diet & . & ** & $\mathrm{VE}$ & VE & $*$ & VE \\
\hline Genetic 1 & $*$ & VE & VE & $\mathrm{VE}$ & VE & $\%$ \\
\hline Treatn & $\mathrm{VE}=4.69 \%$ & $\mathrm{VE}=$ & $\mathrm{VE}=0.74^{\circ}$ & $\mathrm{VE}=0.36 \%$ & $\mathrm{VE}=0.40 \% *$ & $1 \%$ *** \\
\hline Diet ${ }^{*}$ Genetic line & $3 \% * * *$ & $0.96 \%$ & $\mathrm{VE}=2.88 \% * * *$ & $\mathrm{VE}=5.03 \% * * *$ & $\mathrm{VE}=5.09 \% * * *$ & $.52 \%$ \\
\hline Diet*Treatment & $\mathrm{VE}=8.02 \% * * *$ & $\mathrm{VE}=1.41 \% * * *$ & $\mathrm{VE}=0.10 \%$ & $\mathrm{VE}=0.07 \%$ & $\mathrm{VE}=0.45 \%$ & $\mathrm{VE}=3.55 \% * * *$ \\
\hline Genetic line*Trea & $\mathrm{VE}=0.98 \%$ * & $\mathrm{VE}=2.82 \% * * *$ & $\mathrm{VE}=1.06 \% * * *$ & $\mathrm{VE}=1.81 \% *$ & $\mathrm{VE}=3.53 \% * * *$ & $\mathrm{VE}=0.72 \%$ \\
\hline Diet*Treatment ${ }^{*}$ Genetic line & $\mathrm{VE}=2.37 \% * * *$ & $\mathrm{VE}=0.82 \%$ & $\mathrm{VE}=2.75 \% * * *$ & $\mathrm{VE}=4.81 \% * * *$ & $\mathrm{VE}=4.92 \% * * *$ & $\mathrm{VE}=2.37 \%$ \\
\hline
\end{tabular}

Weight: All of the independent variables, with the exception of $\mathrm{D} \times \mathrm{T}$, produced a significant effect on dry larval weight with diet being the best predictor, followed by genotype, and $\mathrm{D} \times \mathrm{G}$ interaction (Table 3 and Supplementary Table S1.5). Triglyceride. Once again, diet explained the largest portion of variance across all independent variables. The interactive effect of $D \times G$ was a better predictor of triglyceride concentrations than the genotype (Table 3 and Supplementary Table S1.5). Protein: In contrast with other measured phenotypes, the variance explained by the model was predominantly evenly distributed across the independent variables, with genotype having the highest predicting power followed by the D $\times$ G interactive effect (Table 3 and Supplementary Table S1.5). Glucose. Diet was the strongest predictor of larvae glucose concentrations (Table 3 and Supplementary Table S1.5). Other variables that produced a significant effect on glucose concentrations were treatment, $\mathrm{D} \times \mathrm{G}$, and $\mathrm{D} \times \mathrm{T}$ (Table 3 and Supplementary Table S1.5).

3.2. The Symbiotic Bacterial Community Composition of the Larvae Raised on the Natural Diet Was Different from the Lab Food Raised Larvae and Was Influenced by Maternally Inherited Bacteria and the Host's Genotype

3.2.1. The Gut Bacterial Community Composition and Diversity Varied Substantially across Dietary and Treatment Conditions

Alpha diversity: We characterized a total of 6763 unique ZOTUs across the whole dataset with the number of ZOTUs per sample ranging from 55 to 886 . The total number of reads per sample ranged from 4685 to 908,308 . Of the ZOTUs that could be assigned a taxonomic classification, we identified 134 classes, 218 families, and 394 genera represented across the samples. The response of alpha diversity to changing diets varied with the larval sterilization treatment. For NS larvae, we found that the Shannon index of larvae raised on PR and R diets was significantly higher than those raised on the PA diet (Supplementary Table S2.1). All other comparisons were not significant. However, if the embryos were subjected to sterilization, the microbial species richness of larvae raised on the PA diet was significantly higher than larvae raised on the PR diet (Supplementary Table S2.1). In addition, there was no significant difference in bacterial species richness between larvae raised on regular or peach regular diets (Supplementary Table S2.1). We observed the exact same pattern for the PD whole tree index. Larvae raised on any diet were not significantly different in Shannon's index (Supplementary Table S2.1).

Beta diversity: The hierarchical clustering of the Bray-Curtis distances indicated that the most distant bacterial communities were formed between larvae raised on the $R$ and PR diet (Figure 2A). This pattern held true for both sterilized and non-sterilized larvae (Figure 2A). Clustering weighted 
Unifrac distances suggested that $P R$ and $R$ diets might produce symbiotic bacterial communities that were phylogenetically distant from each other, especially if the parental microbiota had been removed (Figure 2B).

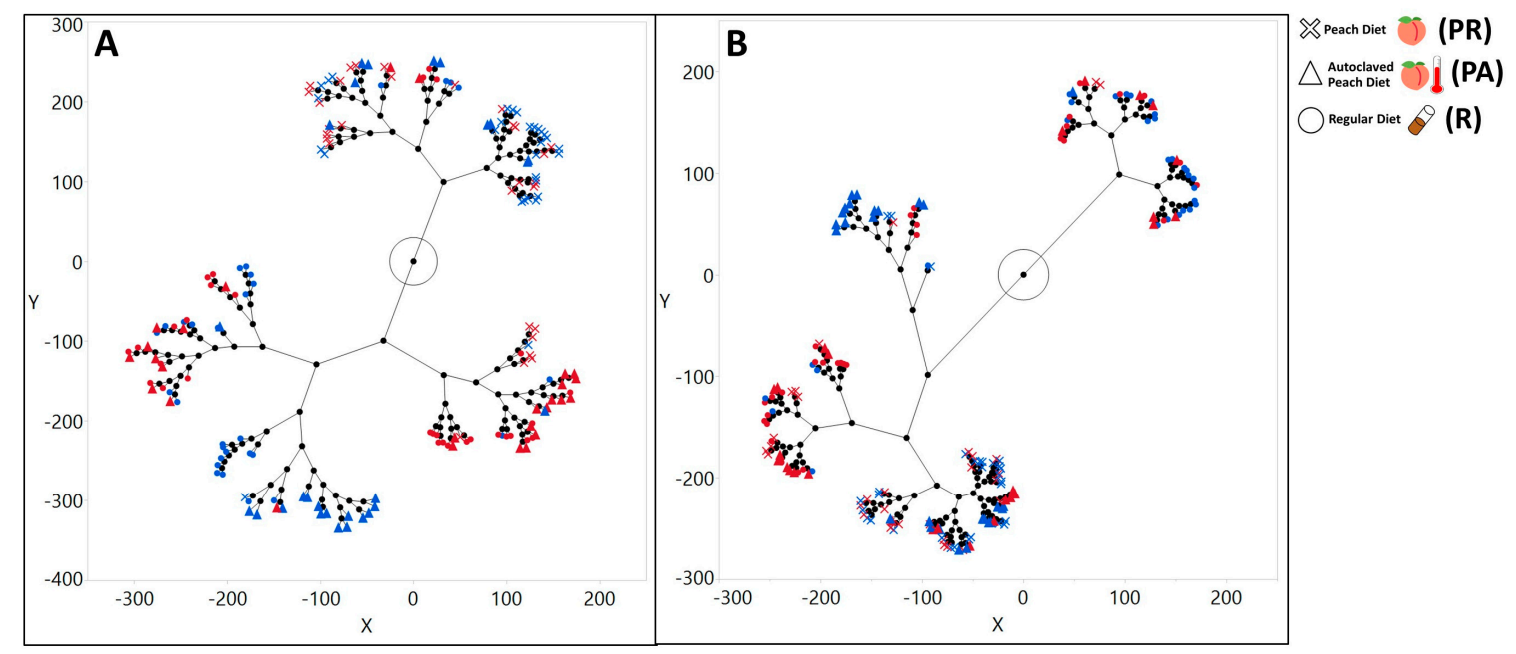

Figure 2. Larvae raised on peach and regular lab diets form distinct bacterial communities. Constellation plot based on hierarchal clustering of larvae bacterial community beta diversity distances. (A) Bray-Curtis distances indicate that symbiotic bacteria of the larvae raised on a peach diet is different from larvae raised on a regular diet. Symbiotic bacterial communities of PA raised larvae are more similar with $\mathrm{R}$ raised larvae than with PR raised larvae, especially if larvae were not sterilized. (B) Weighted Unifrac distances indicate that based on phylogenetic beta diversity distances PR and R diets form distinct communities. Samples from sterilized larvae are marked with a blue color and samples from non-sterilized larvae are marked with the red color. Samples raised on regular lab diet are marked with circled, on a peach diet with crosses and on peach autoclaved diet with triangles.

Taxa composition: Applying discriminant analysis on the ten most abundant bacteria at each taxonomic level revealed which organisms were largely responsible for the differentiation of the bacterial composition on the canonical plot, based on diet. Thus, PR food is largely defined by the abundance of Cyanobacteria at the phylum level (Supplementary Figure S1A,B), Epsilonproteobacteria at the class level (Supplementary Figure S1C,D), Streptophyta at the order level (Supplementary Figure S1E,F), Leuconostocaceae sequences at the family level (Supplementary Figure S2A,B), and Leuconostoc at the genera level (Figure 3A, Supplementary Figure S2C,D). In turn, the lab diet was defined by Firmicutes, Bacilli, Lactobacillales, Lactobacillaceae, and Lactobacillus respectively (Figure 3A, Supplementary Figures S1 and S2). Interestingly, when we considered only the 10 most abundant organisms at each taxonomic level, we did not see a full separation between $\mathrm{R}$ and PA diets unless the larvae were sterilized (Figure 3A, Supplementary Figures S1 and S2). If parental microbiota were removed, the differentiation of the PA diet was led by Actinobacteria at the phylum level, Actinobacteria and Alphaproteobacteria at the class level, Clostridiales and Rickettsiales at the order level, Lachnospiraceae and Rickettsiaceae at the family level, and Bacteroides and Wolbachia at the genus level (Figure 3A, Supplementary Figures S1 and S2). Including all identified bacterial groups in the discriminant analysis revealed that diet was a good predictor of bacterial taxa composition at the phylum, class, order, family, genus, and even individual ZOTU levels (Figure 3B, Supplementary Figures S3 and S4). 


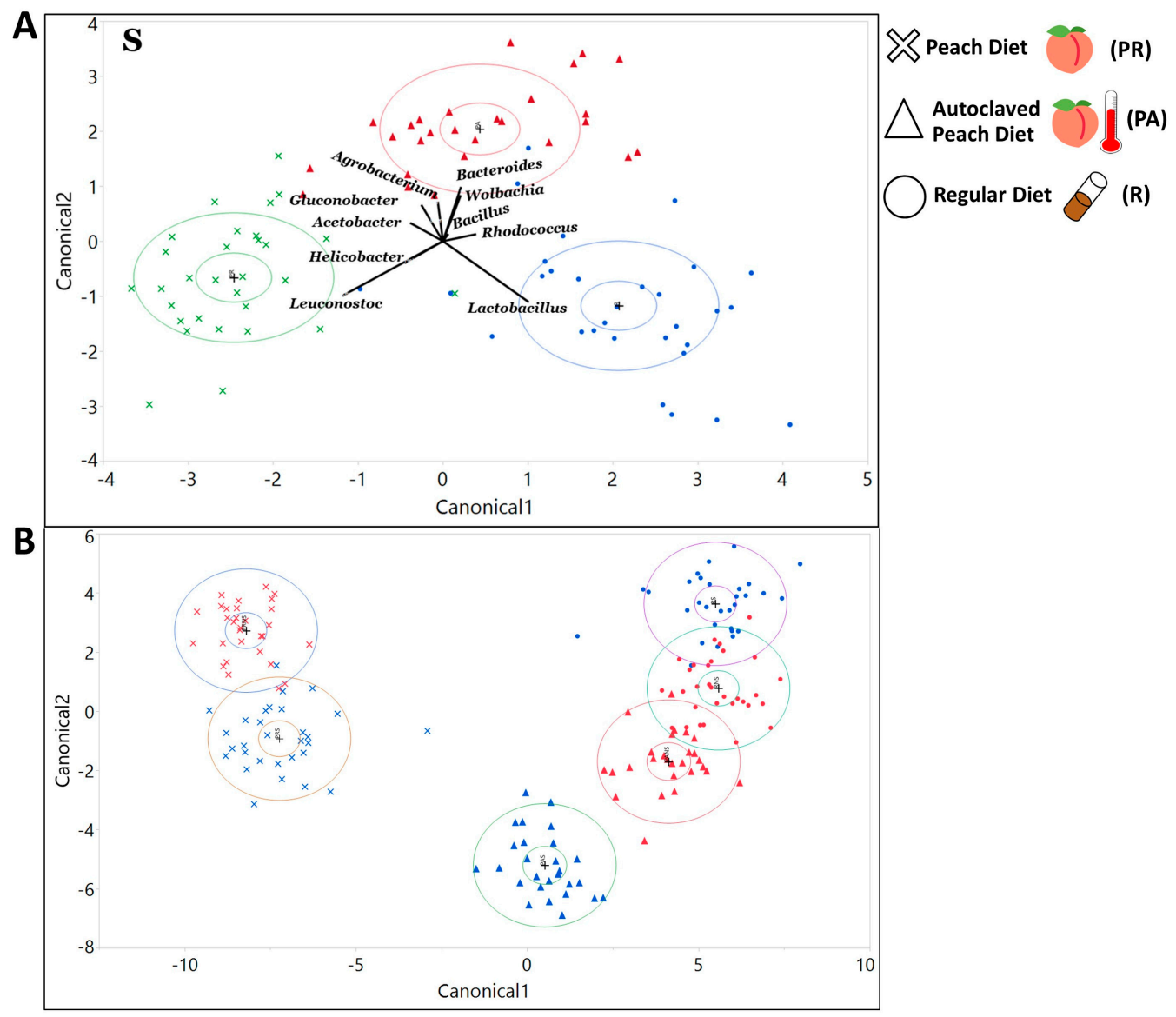

Figure 3. Microbial communities of the larvae raised on all of the diets could be differentiated by the abundance of dominant bacteria taxa. Discriminant analysis of symbiotic bacterial community based on taxa relative abundances in larvae. (A) The 10 most abundant bacterial genera found in sterilized larvae. Diet serves as a good predictor for bacterial communities differentiation. The length of the vector is correlated with the strength of the impact that it produced for the samples to be separated, in the vector direction, on the canonical plot. (B) The 100 most abundant ZOTU found in larvae. All diets and treatments (non-sterilized red and sterilized blue) form distinct communities, with the PR raised larvae being the most different from larvae raised on other diets.

Overall, for non-sterilized larvae the abundance of eight phyla, 12 classes, 20 orders, 27 families, 40 genera, and 141 ZOTUs were significantly different between PR and R food (Supplementary Table S3). Comparing PR and PA food, we found that the abundance of 4 phyla, 6 classes, 9 orders, 16 families, 20 genera, and 76 ZOTUs were significantly different (Supplementary Table S3). Lastly, we observed the significant difference for the abundance of four phyla, three classes, five orders and families, three genera, and 27 ZOTUs between R and PA food (Supplementary Table S3). This indicated the minimal difference between microbial communities of these diets to be consistent with the discriminant analysis.

In larvae lacking parental microbiota, we observed that the abundance of 6 phyla, 10 classes, 18 orders, 34 families, 37 genera, and 114 ZOTUs were significantly different between lab and peach diets (Supplementary Table S3). Comparing PR and PA diets, we found a significant difference in the abundance of seven phyla, 14 classes, 32 orders, 48 families, 67 genera, and 200 ZOTUs (Supplementary Table S3). R and PA diets were significantly different in the abundance of 5 phyla, 6 classes, 9 orders, 13 families, 18 genera, and 87 ZOTUs (Supplementary Table S3). 
3.2.2. The Maternally Transmitted Microbiota Influenced the Composition of the Larvae's Symbiotic Bacterial Communities

Alpha diversity: S larvae had higher values for species richness, Shannon, and PD whole tree indexes on the PA diet (Supplementary Table S2.2). NS larvae had a significantly higher Simpson index value on the PR diet (Supplementary Table S2.2). All other comparisons were not significantly different.

Beta diversity: When comparing the difference between beta diversity metrics in NS and S treatments for each diet, we observed a distinctive clustering, based on the treatment of samples that were raised on PA food for Bray-Curtis distance (Figure 4A) and weighted Unifrac distance (Figure 4D). For the samples that were raised on $\mathrm{R}$ food, we observed the clustering for weighted Unifrac distance only (Figure 4F).
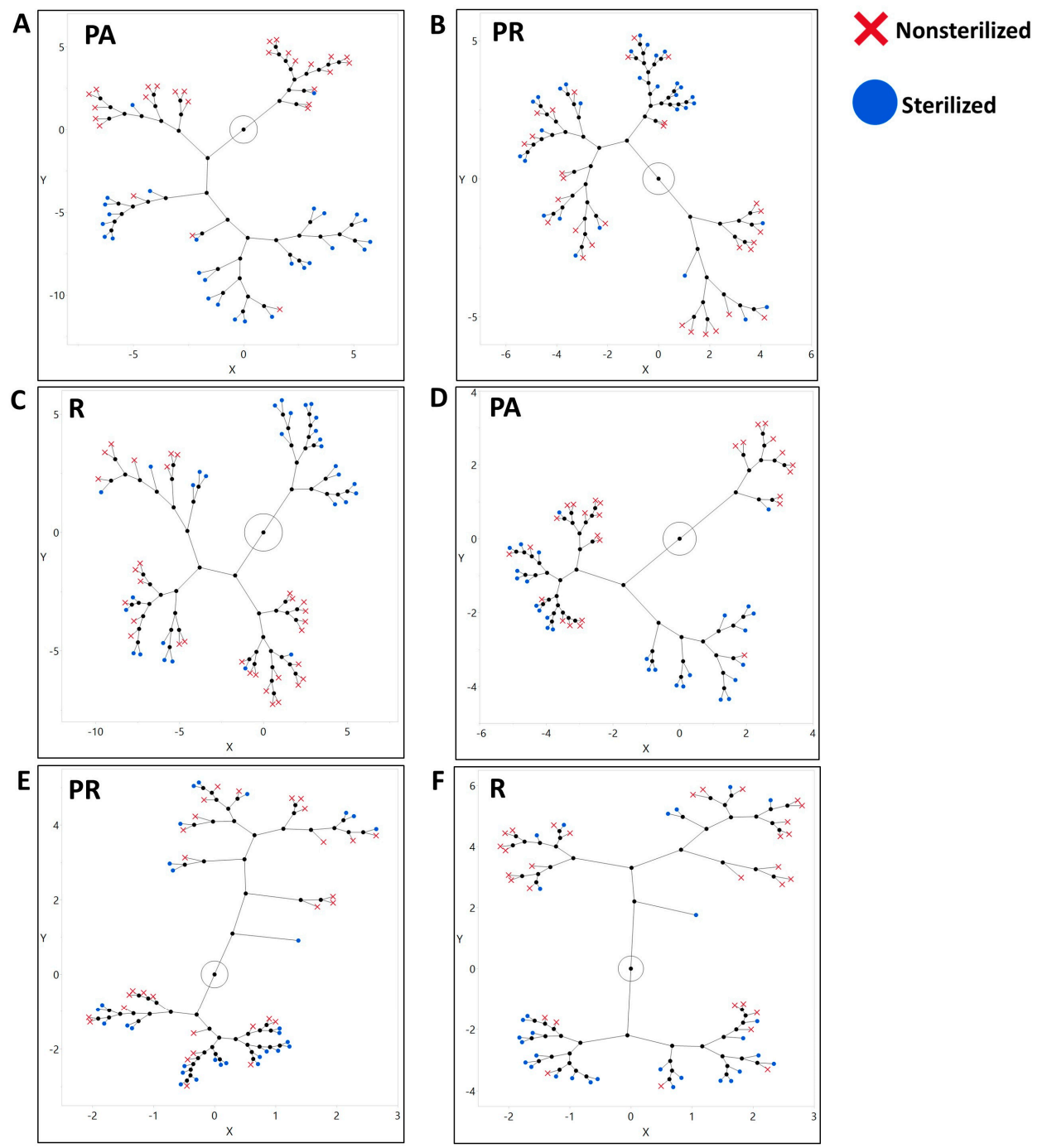

Figure 4. Inheriting parental bacteria influences the formation of larvae symbiotic bacterial community with the effect being unequal among the diets. (A-C) Bray-Curtis distances between NS and S larvae raised on PA, PR, and R diets, respectively. NS and S communities are clearly separated on (A) PA and (C) $R$ diets, and (B) PR food is less distinct. (D-F) Weighted Unifrac distances. Larvae raised on (A) PA and $(C) R$ diets form more distinct communities than larvae raised on (B) PR diet.

Taxa composition: The discriminant analysis indicated that the status of inheritance of the parental bacteria could serve as a good predictor for differentiation of the bacterial community as indicated with the canonical plot on all taxonomic levels (phylum, order, class, family, genus, and ZOTU; Figure 5, Supplementary Figures S5 and S6). Among the 10 most abundant phyla that 
defined the differentiation of the NS community were Firmicutes and Bacterioidetes on the PA diet (Supplementary Figure S7A), Firmicutes on the PR diet (Supplementary Figure S7B), and Proteobacteria, Tenericutes, and Flusobacteria on the R diet (Supplementary Figure S7C). Phyla that were influential for differentiation of the $S$ community were Actinobacteria, Fusobacteria, and Cyanobacteria on the PA diet (Supplementary Figure S7A), Actinobacteria, Tenericutes, and Flusobacteria on the PR food (Supplementary Figure S7B), and Planctomycets and Bacterioides on the R diet (Supplementary Figure S7C). Considering bacterial classes, the NS treatment was strongly defined by Bacilli on the PA (Supplementary Figure S7D), and the PR diets (Supplementary Figure S7E), and Alphaproteobacteria and Bacteroida on the R food diet (Supplementary Figure S7F). The sterilization treatment was mostly separated due to Actinobacteria on the PA diet, Alpha and Beta proteobacteria on the PR diet, and Gammaproteobacteria on the R diet (Supplementary Figure S7D,E). On R food, microbial communities from the sterilized and non-sterilized larvae were not fully separated on the canonical plot Supplementary Figure S7F). At the order level, the NS community was defined by Lactobacillales on the PA and the PR diets, and Actinomycetales and Rhodospirillales on the R food (Supplementary Figure S7G-I). Sterilized larvae were associated with abundances of Streptophyta on the PA food, and Burkholderiales and Rhodospirillales on the PR diet (Supplementary Figure S7G-I). On the $R$ diet, 4 out of 10 tested orders were strongly associated with the S treatment (Supplementary Figure S7I). At the family level, the NS larvae were correlated with Lactobacillaceae on the PA and the PR diets and Acetobacteraceae on the R food diet (Supplementary Figure S8A-C). Sterilized larvae were defined by the abundance of Nocardiaceae on the PA food and Leuconostocaceae and Caulobactereceae on the R diet (Supplementary Figure S8A-C). At the genera level, NS was primarily separated by Lactobacillus on the PA diet and Acetobacter and Agrobacterium on the R food (Figure 5). On the PR food diet, 95\% confidence ellipses almost overlapped, indicating that sterilization status might not be the decisive predictor for the abundance of the 10 most common genera (Figure 5). The $S$ treatment was primarily defined by the abundance of Leuconostoc and Gluconobacter on the PA diet and Lactobacillus and Leuconostoc on the R diet (Figure 5).

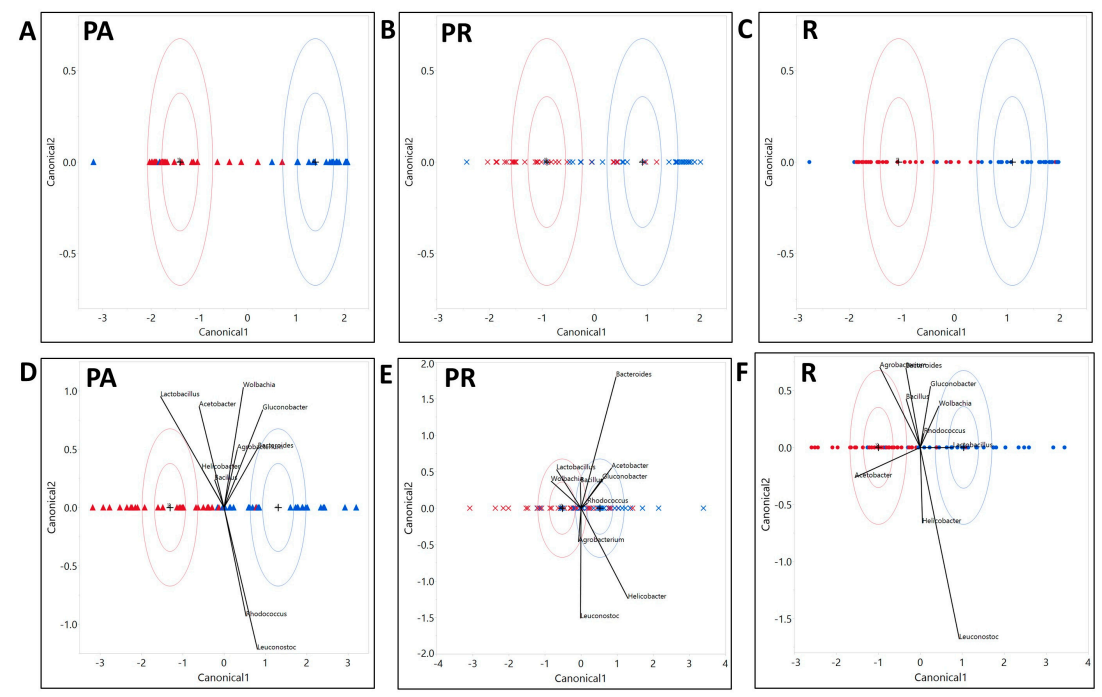

Figure 5. Microbial communities of the larvae raised with or without parental microbiota could be differentiated by the abundance of all bacteria taxa and by the abundance of the dominant taxa only. Discriminant analysis of symbiotic microbiota community based on taxa relative abundances in larvae from non-sterilized (red) and sterilized (blue) treatments. The analysis includes (A) All identified ZOTUs on a PA diet (B) on a PR diet, and (C) on an R diet. On all diets, treatment serves as a good predictor for a differentiation of the symbiotic bacterial communities. (D) The 10 dominant genera on a PA diet. (E) The 10 dominant genera on a PR diet. (F) The 10 dominant genera on an R diet. Treatment serves as a good predictor for the separation of bacterial communities on (D) PA and (F) R diets and is associated with the dominant bacteria taxa. 
Multiple microbial groups were significantly different in their distribution between NS and $S$ treatments across the diets on all taxonomic levels. On regular food, the abundance of 4 phyla, 4 classes, 5 orders, 6 families, 9 genera, and 41 ZOTUs were significantly different (Supplementary Table S4). On the PR diet we saw a significant difference in the abundance of 1 phylum, 2 classes, 6 orders, 9 families, 11 genera, and 61 ZOTUs (Supplementary Table S4). The highest number of significantly different taxa was observed on the PA diet with 7 phyla, 12 classes, 21 orders, 34 families, 43 genera, and 148 ZOTUs (Supplementary Table S4).

3.2.3. The Composition of the Microbial Community Exhibited Variation with Host Genotype, Which Further Exhibited a Significant Interactive Effect with Diet and Treatment

We also tested the influence of genotype and other variables' interactive effect on the abundance of bacteria. At the phyla level, 14 were significantly influenced by genotype, three by $\mathrm{D} \times \mathrm{G}$ interaction, five by $\mathrm{G} \times \mathrm{T}$, five by $\mathrm{D} \times \mathrm{T}$, and six by $\mathrm{D} \times \mathrm{G} \times \mathrm{T}$ (Supplementary Table S5). Abundances of 30 classes were significantly influenced by genotype, eight by $\mathrm{D} \times \mathrm{G}, \mathrm{G} \times \mathrm{T}$, and $\mathrm{D} \times \mathrm{T}$, and 10 by $\mathrm{D} \times \mathrm{G} \times \mathrm{T}$ interaction (Supplementary Table S5). Among the orders, an abundance of 46 was significantly influenced by genotype, 13 by $\mathrm{D} \times \mathrm{G}, 15$ by $\mathrm{G} \times \mathrm{T}, 16$ by $\mathrm{D} \times \mathrm{T}$, and 15 by $\mathrm{D} \times \mathrm{G} \times \mathrm{T}$ (Supplementary Table S5). The abundance of 72 families was significantly influenced by genotype, 20 by $\mathrm{D} \times \mathrm{G}, 15$ by $\mathrm{G} \times \mathrm{T}, 18$ by $\mathrm{D} \times \mathrm{T}$, and 23 by $\mathrm{D} \times \mathrm{G} \times \mathrm{T}$ (Supplementary Table S5). Lastly, genotype significantly influenced 94 genera, $\mathrm{D} \times \mathrm{G}$ influenced 44, $\mathrm{G} \times \mathrm{T}$ influenced $46, \mathrm{D} \times \mathrm{T}$ influenced 30 , and $\mathrm{D} \times \mathrm{G} \times \mathrm{T}$ influenced 45 genera (Supplementary Table S5).

3.3. We Identified Microbial Taxa That Exhibited Correlations with Host Phenotype across Diets and Treatments, with Many that Had a Diet, Treatment, or Genotype Specific Relationship

Across the NS larvae, we found four significant interactions on the R food, 14 on PR diet, and 14 on PA diet at the phylum level (Supplementary Table S6A, Supplementary Table S6B). For the $S$ larvae at the same taxonomic level, we found eight significant interactions on the $R$ diet, five on the PR, and two on PA food (Supplementary Table S6A, Supplementary Table S6B). At the class taxonomic level, 9 significant interactions were found on $\mathrm{R}$ food, 35 on PR, and 24 on PA diet (Supplementary Table S6A, Supplementary Table S6B). Considering S larvae, there were 23 significant correlations on the R diet, 11 on PR, and 4 on the PA diet (Supplementary Table S6A, Supplementary Table S6B). For NS larvae at the order level, we found 23 significant correlations on the $\mathrm{R}$ diet, 57 on PR, and 46 on PA diet (Supplementary Table S6A, Supplementary Table S6B). For S larvae we found 29 significant correlations on R food, 22 on PR, and 9 on PA diet (Supplementary Table S6A, Supplementary Table S6B). At the family level, we found 34 significant correlations on R food, 88 on PR, and 67 on PA diet for NS larvae (Supplementary Table S6A, Supplementary Table S6B). For S larvae, we observed 51 significant interactions on R food, 29 on PR, and 14 on PA diets (Supplementary Table S6). Across the genera, we found 40 significant interactions on R, 105 on PR, and 64 on PA diets, for NS larvae (Figures 6 and 7, Supplementary Table S6A, Supplementary Table S6B). Considering S larvae, we found 76 significant interactions between tested taxa and phenotypes on $R, 46$ on PR, and 33 on PA diets (Figures 6 and 7, Supplementary Table S6A, Supplementary Table S6B). At the level of individual ZOTUs, for NS larvae, we found 226 significant interactions on R, 283 on PR, and 225 on PA diets (Supplementary Table S6A, Supplementary Table S6B). For S larvae the number of significant interactions between ZOTUs abundances and larvae phenotypes were as follow 313 on R, 164 on PR, and 230 on PA diets (Supplementary Table S6A, Supplementary Table S6B). 

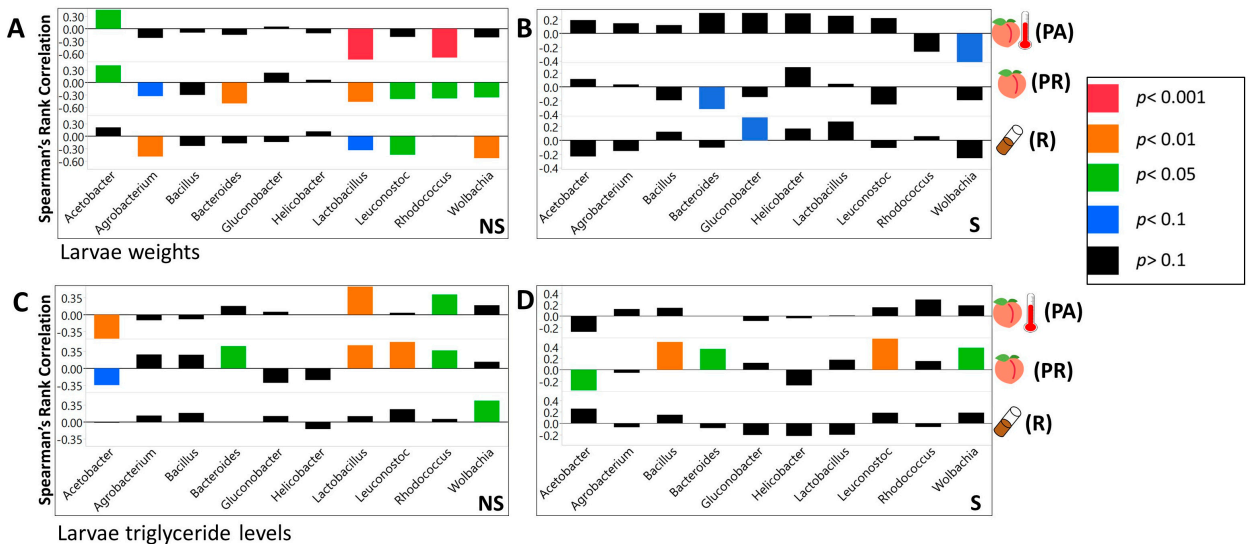

Larvae triglyceride levels



Figure 6. The correlations between the abundance of symbiotic bacterial taxa and larvae metabolic phenotypes vary with the diets. Spearman's rank correlation coefficients between the abundance of 10 dominant bacterial genera and larvae phenotypes, on each diet. The color of the bars corresponds to the level of significance for each correlation (uncorrected for multiple comparisons). (A) Weight of NS larvae. (B) Weight of S larvae. (C) Triglyceride levels of NS larvae. (D) Triglyceride levels of S larvae. (E) Glucose levels of NS larvae. (F) Glucose levels of S larvae. The effect that symbiotic bacteria produce on all metabolic phenotypes may change with the diet.
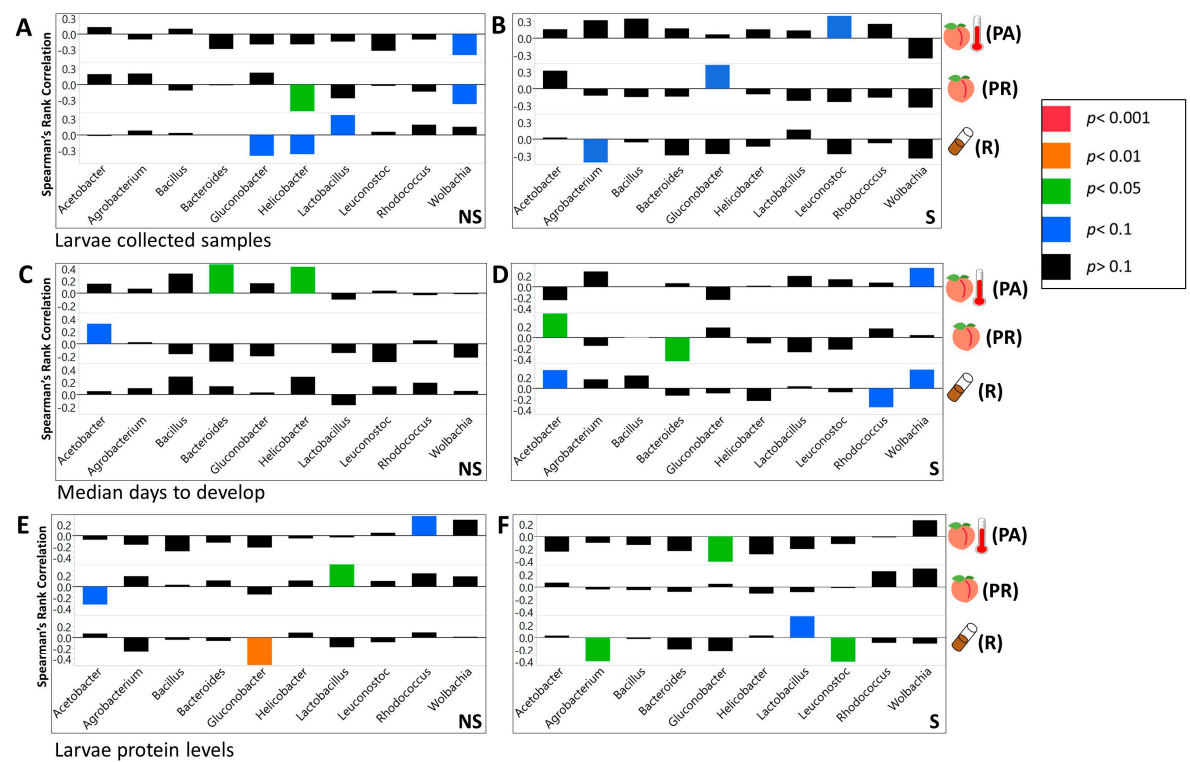

Figure 7. The correlations between the abundance of bacterial taxa and larvae life history traits and metabolic phenotypes vary with the larval diet. Spearman's rank correlation coefficients between the abundance of 10 dominant symbiotic bacterial genera and larvae phenotypes on each diet. The color of the bars corresponds to the level of significance (uncorrected for multiple comparisons) for each correlation. (A) Total proportion of NS larvae reaching the 3rd instar stage; (B) total proportion of $S$ larvae reaching the 3 rd instar stage; (C) median number of days to reach pre-pupation stage for NS larvae; (D) median number of days to reach prepupation stage for S larvae; (E) protein concentrations of NS larvae; and (F) protein concentrations of S larvae. Symbiotic bacteria may influence life history traits and protein concentrations of the larvae differently depending on a diet. 
We evaluated the interactive effect of the abundance of microbial taxa and diet, genotype, and treatment on forming the tested phenotypes. $\mathrm{D} \times \mathrm{A}$ produced a significant effect in 11 cases at the phylum level, in 26 at the class level, in 52 at the order level, in 72 at the family level, and in 95 cases at the genus level (Supplementary Table S7). We found a significant G $\times$ A interaction in eight cases at the phylum level, in 10 at the class level, in 39 at the order level, in 57 at the family level, and in 105 cases at the genus level (Supplementary Table S7). $\mathrm{T} \times$ A produced a significant effect in 13 cases at the phylum level, in 27 at the class level, in 36 at the order level, in 60 at the family level, and in 87 cases at the genus level (Supplementary Table S7).

\section{Discussion}

4.1. Frozen Peach Food Was Capable of Providing Nutritional Conditions Similar to the Natural Ones and Can Preserve Key Microbial Taxa Necessary for Survival and Development of Drosophila Larvae

The reduction in survival, increase in development time, and increase in triglyceride concentrations, and decreased weight and protein concentrations of the larvae raised on the natural food compared with the larvae raised on the $\mathrm{R}$ lab food resembles the phenotype generated by a reduced protein diet. These findings correlate with our evaluation of the protein concentrations in different diets [49-52]. In addition, the adaptation of Drosophila to the lab environment was connected to increased weight and reduced stress tolerance [53-55]. Therefore, nutritional and pathogenic stresses associated with the natural food conditions could further contribute to the decrease in survival and development rate of larvae raised on the PR food compared to the standard diet $[50,52,56,57]$.

The pattern regarding glucose concentration was more interesting. Freshly unfrozen peach food had a higher glucose concentration than regular lab food, but larvae raised on the PR diet had the lowest concentration compared to larvae raised on any other diet. This pattern was likely caused by the activity of naturally acquired microbes since it was shown that the presence of several microbial taxa that naturally associate with Drosophila, such as Acetobacter, is correlated with decreased sugars in fly food and Drosophila itself $[14,15]$. In addition, incubation of the PR food, even without the larvae, led to a drastic reduction of glucose concentrations compared to the $\mathrm{R}$ and PA diets.

It is well known that heat application can result in the decomposition of sugars and other macronutrients $[58,59]$. However, the difference between all phenotypes (with the exception of glucose) increased if the peach food diet was autoclaved and even more (with the exception of triglyceride) if the parental microbiota were not transferred to the autoclaved diet. This suggested that symbiotic bacteria played an important role in shaping the phenotypic change between PR and PA raised larvae. These findings are consistent with previous studies that showed that the presence of naturally associated bacteria was advantageous for Drosophila melanogaster and Drosophila suzukii on fresh fruit diets, which are also poor in protein content [50,57]. In fact, larvae raised on a PA diet closely resembled the phenotype of axenic larvae and axenic larvae raised under low protein nutritional conditions. Examples of this similar phenotype include lower survival and body size/weight [12,14,23], longer development time [13,23], and elevated glucose [15] and triglyceride concentrations [13,14]. Although, larvae raised on the PA diets exhibited significantly longer development time than on any other diet, we did observe minimal changes in the PA food nutrient concentrations during the incubation period (Supplementary Table S1.2, Supplementary Table S1.3). Therefore, it is unlikely that larval metabolic phenotype would be influenced by depletion of nutrients during these extra days.

\subsection{Maternally Deposited Microbes Produced Positive Effects on Larvae that Were Raised on the Peach Diets}

Interestingly, the presence of parental microbiota did not produce a significant effect on any of the tested phenotypes, when larvae were raised on the lab diet. Contrarily, on the peach diet, the presence of parental microbiota increased the weight and development rate even if the original peach microbiota were still present. These findings are consistent with the reports of beneficial effects, of the maternally deposited microbiota, for larvae on a fruit diet. These results also indicate the 
importance of considering an organism's natural environmental conditions when addressing the questions about symbiotic relationships and evolutionary patterns [50,57].

\subsection{Genotype Was One of the Key Factors that Influenced Larvae Phenotypes}

It is important to note that although the described patterns were observed for the total experimental population of larvae, the genetic component still played a significant role in generating all but the glucose phenotype. In addition, consistent with previous research, we observed that $D \times G$ interaction played a significant role in forming the metabolic phenotype and contributed to the survival of the organism [16,36]. Furthermore, most of the tested phenotypes were significantly correlated with $\mathrm{G} \times \mathrm{T}$ and even $\mathrm{D} \times \mathrm{G} \times \mathrm{T}$, indicating the importance of considering multiple factors to understand the development of complex traits.

\subsection{Bacteria of the Larvae Raised on PR Food Exhibit a Distinct Community Structure}

Multiple studies were performed to evaluate the gut microbiota composition of lab and wild populations of Drosophila [24,57,60-62]. Although most of them consistently report the prevalence of different members of Alphaproteobacteria, Bacilli, or Gammaproteobacteria in lab and wild populations, the relative abundance of the taxa, especially at lower taxonomic levels, often varies between studies [61]. In our work, larvae raised on the PR food diet formed a distinct community clearly separated from the larvae raised on the $\mathrm{R}$ food diet, as displayed on the canonical plot. We observed a higher prevalence of Gluconobacter and Leuconostoc and lower abundance of Lactobacillus in larvae raised on the PR diet compared with the $\mathrm{R}$ food diet $[24,56,57,63]$, which is consistent with previous findings performed on natural populations of Drosophila.

However, it is difficult to judge how well the microbial community of our experimental larvae represents the microbial community of wild population, as a variety of factors could influence gut microbiota composition in flies, which certainly complicates comparisons between studies $[56,61,64]$. As such, it was shown that the gut microbial composition of lab reared flies might vary with diet (and even among the standard diets with the major carbohydrate source), genetic line, development stage temperature, and etc. [61,64-66]. The wild populations of gut microbiota in Drosophila were shown to vary with collection location and diet $[56,60,62,67]$. In other insects and wild populations of vertebrates, gut microbiota was shown to change even with seasonality [68-71].

In addition, the relationship between Drosophila gut microbiota during the developmental and adult stages is a subject of controversy between a few studies that compared those relationships $[25,64,65]$. Furthermore, to the best of our knowledge, the gut bacteria of the larvae from the natural populations were not assessed at all. This is likely due to the complexity of identifying Drosophila species during the larval stage. Therefore, we hope to provide the methodology for the possibility of exploring the effects of a natural diet, and the microbial community associated with it, in a controlled lab environment. This setting provides the opportunity to work not only with adult flies but also with larvae.

\subsection{Community Structure of Symbiotic Bacteria Were Correlated with Diet, Treatment, Host Genotype, and Their Specific Interactive Effects}

The development of symbiotic microbiota populations was shown to be correlated with the available nutrients present in the diet, the host's genotype, and parental microbiota left on the chorion of the egg $[12,61,64]$. Complementary to the results reported by Jehrke [64], we also observed that the genotype of the host may influence the abundance of bacterial taxa more than the diet. Wong [12] reported that the bacterial population deposited on the Drosophila embryo may shift the symbiotic microbiota population of the offspring, even in the presence of bacteria that previously colonized the food substrate. We observed similar results in most cases.

However, among the 10 most abundant genera on the PR diet, the full separation of the $\mathrm{S}$ and NS larvae microbial community compositions was not present on the canonical plot indicating the possibility of a difference in the response of the lab and the natural microbial population to the presence 
of Drosophila parental bacteria. This differentiation was not likely caused by the nutrition composition of the food since the PA separation, represented on the canonical plot, between S and NS treatments was obvious in all cases. In addition, for beta diversity distances, the abundance of individual microbial taxa, and the correlations between the abundances of microbial taxa within the microbial community, represented patterns found in PA food that resembled the ones in the R food raised larvae, if parental microbiota were not removed (Figures 2 and 3, Supplementary Figure S9). Overall, consistently with previous studies, our findings indicated the dependency of relative bacterial abundances on all of the tested variables and demonstrated the interactive effect between these variables [12,61,64].

\subsection{The Correlations between Microbial Taxa as Well as the Correlation between the Whole Microbial Community and the Host May Vary with the Diet and Other Environmental and Genetic Conditions}

Genotype and gut microbiota composition are among the major factors that control the development of obesity traits [72]. Changes in some key microbiota populations are associated with the rapid expansion in the prevalence of metabolic syndrome [73]. Alterations in the gut microbiota community can modulate insulin secretion and sensitivity, thus contributing to diabetes susceptibility [74]. Moreover, previous research indicates that genetic variation considerably influences the gut microbiota composition $[64,73,74]$. However, most of the studies mentioned above have used less than ten genotypes to study the correlation between gut microbiota and the pathogenesis of obesity in mice. The challenges of using a mouse model involve relatively high expenses for husbandry and logistics [75-77].

Drosophila melanogaster is an exceptional model to study the effect of genotype on the phenotype formation, due to the variety of established tools such as Drosophila Genetic Reference Panel and The Drosophila Synthetic Population Resource. These resources offer a variety of diverse genotypes, with sequenced parental genomes, that allow for testing the microbiota effects across various genetic backgrounds and provides potential for studying genetic interaction between the host and its symbionts, and even mapping the specific genetic loci responsible for the interactions [21,31,78]. The phenotypic response to a diet modification often varies with the genotype [16,36]. In fact, diet-by-genotype $(D \times G)$ interaction may explain more variance than diet alone in the metabolic response of such traits, such as triglyceride and carbohydrate concentrations [36]. In addition, recent findings showed that genotype-by-diet interactions significantly influences metabolomic profiles; hence, laying the foundation for explaining the mechanism through which $\mathrm{D} \times \mathrm{G}$ influences metabolic traits [16,79]. Our results are consistent with previous research, in that phenotypic response varied significantly between genetic lines [16,79]. Genotype had a significant effect on survival, development rate, and triglyceride concentrations, and was the second-best predictor of weight and the best predictor of protein concentrations.

Obesity and type two diabetes are associated with elevated weight, high blood glucose concentrations, and excess accumulation of adipose tissue [80,81]. Consistent with recent studies linking Lactobacillus and Coprococcus to obesity in humans, our results show that these genera are positively associated with glucose concentrations [82-85]. In addition, previous research has shown an overall decrease in the abundance of Firmicutes in obese humans [86]. Similarly, we observed that the total abundance of Firmicutes is negatively associated with triglyceride concentrations. It should be noted that the correlation between metabolic phenotype and particular microbial taxa could vary between studies $[5,87,88]$.

Consistently with previous Drosophila research, we observed that the abundance of microbial taxa was correlated with measured phenotypes. Acetobacteraceae was negatively correlated with larval glucose concentrations [20,21,61]. Additionally, Acetobacter was positively correlated with development time while Lactobacillus and Firmicutes were negatively correlated with it [20,21,89]. Previous work showed that Acetobacter species reduced triglyceride concentrations while most Lactobacillus species had no effect $[13,21]$. In contrast, our data shows that Acetobacter was not significantly correlated with triglyceride concentrations, and Lactobacillus showed a negative correlation. Consistent with 
Newell and Douglas [13], we found that L. brevis and L. plantarum were not significantly correlated with protein concentrations, but in addition, our results indicated that Acetobacter abundance was negatively correlated with protein concentrations.

Consistent with Jehrke [64], we observed that most of the correlations between the tested phenotype and abundance of bacteria were relatively weak. Weaker correlations observed with large sample sizes in microbiome research, while significant, failed to hold up to the use of stricter FDR values or other conservative $\mathrm{p}$ adjustment methods [47,64]. Expanding the analysis of the bacterial species abundance for each phenotype beyond the most dominant species, while providing a more complete overview of the correlation between tested phenotypes and microbial abundance, also raises FDR values as a result of increasing sample sizes [47]. Previous microbiome studies have dealt with high FDR values by accepting higher thresholds, so as to not miss possible correlations [47]. Since the level of FDR that should be tolerated is poorly defined and often widely variable compared to accepted $p$-values, its value is often seen as arguable [90]. Considering the large sample sizes used in our analysis, using a low FDR value may obscure important correlations between the tested phenotypes and their abundance of microbiota [47].

Some of the inconsistencies between our work and previous studies on the correlation of the abundance of microbial taxa and measured metabolic phenotype perhaps may be addressed to the interactive effect between the variables included in the experiments. Several studies showed that the contribution of the symbiotic microbiota to the host might be observed only in a diet dependent manner. For example, Shin et al. [23] showed that axenic Drosophila larvae would not be able to develop on a protein poor diet without activation of the insulin signaling pathway by its symbiotic microbes. Wong et al. [19] found diet-dependent differences in microbiota produced effects, including reduction of vitamin requirements on a low-yeast diet and suppression of lipid and carbohydrate storage on a high-sugar diet. Bing et al. [50] found that symbiotic microbiota of D. suzukii are critical for providing proteins for development of flies raised on fresh fruit, but that these microbial proteins are not essential for development of flies raised on a nutrient sufficient diet.

In our study, we also observed that the effect of bacterial abundance produced at the level of individual taxa on larvae phenotype varied with diet. In a few cases, even the direction of correlation between the abundance of microbial taxa and the tested phenotype was opposite on different diets. In addition, using PCA, we observed that correlational effects microbial abundance (as an example at the family level) produced on measured metabolic (Supplementary Figure S10) and fitness phenotypes (development rate and survival; Supplementary Figure S10) varied between the diets. The correlation coefficients for the influence of all microbial taxa on measured metabolic phenotypes clustered together for the PR diet but not for other diets (Supplementary Figure S10). The correlation coefficients between bacteria abundances and fitness phenotypes clustered for all but PA diets (Supplementary Figure S10).

\section{Conclusions}

In conclusion, our work has demonstrated that the bacterial community within a larva is influenced by the parental bacterial community, bacterial community found in the diet, the diet itself, and the genotype of the host. Further, those bacterial communities correlated with host metabolic phenotypes both at the level of specific taxa and as a function of diversity of the community.

Supplementary Materials: The following are available online at http://www.mdpi.com/2076-2607/8/12/1972/s1, Supplementary Figure S1: Bacterial communities of the larvae raised on all of the diets could be differentiated by the abundance of dominant bacteria phyla, classes, and orders. Discriminant analysis of symbiotic bacterial communities based on taxa relative abundances. (A) The 10 most abundant microbial phyla found in non-sterilized larvae, (B) 10 most abundant bacterial phyla found in sterilized larvae, (C) 10 most abundant bacterial classes found in non-sterilized larvae, (D) 10 most abundant bacterial classes found in sterilized larvae, (E) 10 most abundant bacterial orders found in non-sterilized larvae, and (F) 10 most abundant bacterial orders found in sterilized larvae. Diet serves as a good predictor for differentiation of associated bacterial communities. The length of the vector is correlated with the strength of the impact that it produced for the samples to be separated, in the vector direction, on the canonical plot. ZOTUs identified as chloroplast at the class level (C) and (D) are likely to belong to unknown taxa of Cyanobacteria as indicated by the results at phylum taxonomic level (A) and (B). A separate BLAST 
search performed on those sequences did not result in matches to any known eukaryotic chloroplasts as might be expected if they were derived from the diet ingredients (e.g., corn or peach). Supplementary Figure S2: Bacterial communities of the larvae raised on all of the diets could be differentiated by the abundance of dominant bacteria families and genera. Discriminant analysis of symbiotic bacterial community based on taxa relative abundances in larvae. (A) The 10 most abundant bacterial families found in non-sterilized larvae, (B) 10 most abundant bacterial families found in sterilized larvae, (C) 10 most abundant bacterial genera found in non-sterilized larvae, and (D) 10 most abundant bacterial genera found in sterilized larvae. Supplementary Figure S3: Bacterial communities of the larvae raised on all of the diets could be differentiated by the abundance of all identified bacteria phyla, classes, and orders. Discriminant analysis of symbiotic bacterial community based on taxa relative abundances in larvae. (A) All identified bacterial phyla found in non-sterilized larvae. (B) All identified bacterial phyla found in sterilized larvae. (C) All identified bacterial classes found in non-sterilized larvae. (D) All identified bacterial classes found in sterilized larvae. (E) All identified bacterial orders found in non-sterilized larvae. (F) All identified bacterial orders found in sterilized larvae. Diet serves as a good predictor for bacterial communities differentiation. Supplementary Figure S4: Bacterial communities of the larvae raised on all of the diets could be differentiated by the abundance of all identified bacteria families, genera, and ZOTUs. Discriminant analysis of symbiotic bacterial community based on taxa relative abundances in larvae. (A) All identified bacterial families found in non-sterilized larvae. (B) All identified bacterial families found in sterilized larvae. (C) All identified bacterial genera found in non-sterilized larvae. (D) All identified bacterial genera found in sterilized larvae. (E) All identified bacterial ZOTUs found in non-sterilized larvae. (F) All identified bacterial ZOTUs found in sterilized larvae. Diet serves as a good predictor for bacterial communities differentiation. Supplementary Figure S5: Microbial communities of the larvae raised with or without parental bacteria could be differentiated by the abundance of all bacterial phyla, classes, and orders. Discriminant analysis of symbiotic bacterial community based on taxa relative abundances in larvae from non-sterilized (red) and sterilized (blue) treatments. The analysis includes all identified phyla (A) on a PA diet (B) on a PR diet, and (C) on a R diet, all identified classes (D) on a PA diet, (E) on a PR diet, and (F) on a R diet, and all identified orders (G) on a PA diet, (H) on a PR diet, and (I) on a $\mathrm{R}$ diet. On all diets, treatment serves as a good predictor for a differentiation of the symbiotic bacterial communities. Supplementary Figure S6: Microbial communities of the larvae raised with or without parental microbiota could be differentiated by the abundance of all bacteria families, genera, and ZOTUs. Discriminant analysis of symbiotic bacterial community based on taxa relative abundances in larvae from non-sterilized (red) and sterilized (blue) treatments. The analysis includes all identified families (A) on a PA diet, (B) on a PR diet, and (C) on a R diet, all identified genera (D) on a PA diet, (E) on a PR diet, and (F) on a R diet, and all identified ZOTUs $(\mathrm{G})$ on a PA diet, $(\mathrm{H})$ on a PR diet, and (I) on a R diet. On all diets, treatment serves as a good predictor for a differentiation of the symbiotic bacterial communities. Supplementary Figure S7: Microbial communities of the larvae raised with or without parental microbiota could be differentiated by the abundance of dominant bacteria phyla, classes, and orders. Discriminant analysis of symbiotic bacterial community based on taxa relative abundances in larvae from non-sterilized (red) and sterilized (blue) treatments. The analysis includes 10 dominant phyla (A) on a PA diet, (B) on a PR diet, and (C) on a R diet, 10 dominant classes (D) on a PA diet, (E) on a PR diet, and (F) on a R diet, and 10 dominant orders $(\mathrm{G})$ on a PA diet, $(\mathrm{H})$ on a PR diet, and (I) on a R diet. Although on all diets, treatment serves as a good predictor for a differentiation of the symbiotic bacterial communities, it is especially influential on PA food. Supplementary Figure S8: Microbial communities of the larvae raised with or without parental microbiota could be differentiated by the abundance of dominant bacteria families and genera. Discriminant analysis of symbiotic bacterial community based on taxa relative abundances in larvae from non-sterilized (red) and sterilized (blue) treatments. The analysis includes 10 dominant families (A) on a PA diet, (B) on a PR diet, and (C) on an R diet and 10 dominant genera (D) on a PA diet, (E) on a PR diet, and (F) on a R diet. Although on all diets, treatment serves as a good predictor for a differentiation of the symbiotic bacterial communities, it is especially influential on PA food. Supplementary Figure S9: The interaction between microbial genera varies between diets and treatments. The network plot of spearman rank correlation between abundances of 10 dominant bacterial genera (A) from NS larvae on a PA diet, (B) from NS larvae on a PR diet, (C) from NS larvae on a R diet, (D) from $S$ larvae on a PA diet, (E) from S larvae on a PR diet, and (F) from $S$ larvae on a $R$ diet. Microbial correlations of non-sterilized larvae on PA diet are more similar with the R diet than with PR raised larvae. Red lines indicate negative correlation and blue lines indicate positive correlation. The density of the color is positively correlated with the strength of the correlation. The correlations less than 0.5 were filtered out. Supplementary Figure S10: The effect of the whole bacterial communities on larvae metabolic phenotypes and life history traits varies with the diet. (A) Principal component analysis of correlation coefficients between bacterial families and metabolic phenotype of the sterilized larvae from 10 genetic lines. R diet is marked with circles, PR diet is marked with crosses, and PA diet is marked with triangles. Larvae glucose concentration is marked with red, protein is marked with green, triglyceride is marked with blue, and weight is marked with brown colors. (B) Principal component analysis of correlation coefficients between bacterial families and life history of the sterilized larvae from 10 genetic lines. R diet is marked with circles, PR diet is marked with crosses, and PA diet is marked with triangles. Larvae development rate is marked with red and survival is marked with blue. Supplementary file 1 (in text S1.1-1.5): The influence of diet, genotype, treatment, and their interactive effects on larval phenotype. (1.1) The comparison of phenotypes of the larvae raised on R, PR, and PA diets for each genotype (1.2) The difference in nutrient values between fresh diets (1.3) The difference in nutrient values between incubated diets (1.4) The comparison of larval phenotypes raised with (NS) and without (S) parental microbiota. (1.5) The effect produced by diet, treatment, and their interactive effect on larval phenotypes for each genetic line. Supplementary file 2 (in text S2.1-2.2): The comparison of alpha diversity indices between diets and treatments. (2.1) Difference in alpha diversity indices between larvae raised on R, PR, and PA diets. 
Diet in parenthesis has a higher value (2.2). Difference in alpha diversity indices between larvae raised with (NS) and without (S) parental microbiota. Treatment in parenthesis has higher value. Supplementary file 3 (in text S3.1-3.6): The difference in abundances of microbial taxa identified in larvae raised on R, PR, and PA diets. (3.1) Phylum taxonomic rank. (3.2) Class taxonomic rank. (3.3) Order taxonomic rank. (3.4) Family taxonomic rank. (3.5) Genus taxonomic rank. (3.6) Level of individual ZOTUs. Computations were done with Wilcoxon's analysis. Supplementary file 4 (in text S4.1-4.6): The difference in abundances of microbial taxa identified in larvae raised with parental microbiota (NS) and without parental microbiota (S) treatments. (4.1) Phylum taxonomic rank. (4.2) Class taxonomic rank. (4.3) Order taxonomic rank. (4.4) Family taxonomic rank. (4.5) Genus taxonomic rank. (4.6) Level of individual ZOTUs. Computations were done with Wilcoxon's analysis. Supplementary file 5 (in text S5.1-5.5). Influence of diet, genotype, treatment, and their interactive effects on the abundance of microbial taxa. (5.1) Influence on microbiota phyla. (5.2) Influence on microbiota classes. (5.3) Influence on microbiota orders. (5.4) Influence on microbiota families. (5.5) Influence on microbiota genera. Supplementary file 6A (in text S6.1-6.6). The correlations between microbial taxa abundances and larval phenotypes. (6.1) Spearman's rank correlation between larval phenotypes and microbiota phyla. (6.2) Spearman's rank correlation between larval phenotypes and abundance of microbiota classes. (6.3) Spearman's rank correlation between larval phenotypes and abundance of microbiota orders. (6.4) Spearman's rank correlation between larval phenotypes and abundance of microbiota families. (6.5) Spearman's rank correlation between larval phenotypes and abundance of microbiota genera. (6.6) Spearman's rank correlation between larval phenotypes and abundance of microbiota ZOTUs. Supplementary file 6B: The summary of the number of significant interactions between abundance of microbial taxa and larval phenotypes. Sum of the significant spearman's rank correlation between larval phenotypes and bacterial taxa abundances. Supplementary file 7 (in text S7.1-7.15). The influence of interactive effects between abundance of microbiota taxa and diet, genotype, and treatment on larval phenotype. (7.01) Interaction effect between the abundance of microbiota phyla and diet on larval phenotypes. (7.02) Interaction effect between the abundance of microbiota classes and diet on larval phenotypes. (7.03) Interaction effect between the abundance of microbiota orders and diet on larval phenotypes. (7.04) Interaction effect between the abundance of microbiota families and diet on larval phenotypes. (7.05) Interaction effect between the abundance of microbiota genera and diet on larval phenotypes. (7.06) Interaction effect between the abundance of microbiota phyla and host's genotype on larval phenotypes. (7.07) Interaction effect between the abundance of microbiota classes and host's genotype on larval phenotypes. (7.08) Interaction effect between the abundance of microbiota orders and host's genotype on larval phenotypes. (7.09) Interaction effect between the abundance of microbiota families and host's genotype on larval phenotypes. (7.10) Interaction effect between the abundance of microbiota genera and host's genotype on larval phenotypes. (7.11) Interaction effect between the abundance of microbiota phyla and sterilization treatment on larval phenotypes. (7.12) Interaction effect between the abundance of microbiota classes and sterilization treatment on larval phenotypes. (7.13) Interaction effect between the abundance of microbiota orders and sterilization treatment on larvae phenotypes. (7.14) Interaction effect between the abundance of microbiota families and sterilization treatment on larval phenotypes. (7.15) Interaction effect between the abundance of microbiota genera and sterilization treatment on larval phenotypes.

Author Contributions: Conceptualization, A.B. and L.R.; methodology, A.B., S.B. and L.R.; formal analysis, A.B. and S.B.; investigation, A.B., O.C., K.E., S.B., A.R., M.S., A.M., R.C. and L.R.; resources, L.R.; writing-original draft preparation, A.B.; writing-review and editing, A.B., O.C., K.E., S.B., A.R., M.S., A.M., R.C. and L.R.; visualization, A.B.; supervision, A.B. and L.R.; project administration, A.B. and L.R.; funding acquisition, A.B. and L.R. All authors have read and agreed to the published version of the manuscript.

Funding: This research was funded by National Institute for Health (NIH) R01GM098856 to LKR, National Science Foundation (NSF) DEB 1737869 to LKR, Center for Clinical and Translational Research Grant to AB and LKR, University of Alabama System Collaborative Grant to LKR.

Acknowledgments: We appreciate the research assistance of L. Griffin, C. Hart, V. Oza, C. Scott, R. O’Rourke K. MacIntyre, K. Lowman C. Tunckanat, J. Jarnigan, Y. Nam, and all of the members of the Reed lab. Helpful guidance was provided by J. Yoder, J. Olson, S. Chtarbanova-Rudloff, C. Morrow, and J. Lopez-Bautista. Special thanks for research assistance, guidance, and moral support to S. Yan. Funding sources included University of Alabama Graduate School, Department of Biological Sciences, Graduate Student Association, University of Alabama at Birmingham Research Voucher Program, National Institutes of Health: 5R01GMO98856.

Conflicts of Interest: The authors declare no conflict of interest.

\section{References}

1. Rosenberg, E.; Sharon, G.; Atad, I.; Zilber-Rosenberg, I. The evolution of animals and plants via symbiosis with microorganisms. Environ. Microbiol. Rep. 2010, 2, 500-506. [CrossRef] [PubMed]

2. Bordenstein, S.R.; Theis, K.R. Host Biology in Light of the Microbiome: Ten Principles of Holobionts and Hologenomes. PLoS Biol. 2015, 13, e1002226. [CrossRef] [PubMed]

3. Read, M.N.; Holmes, A.J. towards an integrative Understanding of Diet-Host-Gut Microbiome interactions. Front. Immunol. 2017, 8, 538. [CrossRef] [PubMed] 
4. Leitão-Gonçalves, R.; Carvalho-Santos, Z.; Francisco, A.P.; Fioreze, G.T.; Anjos, M.; Baltazar, C.; Elias, A.P.; Itskov, P.M.; Piper, M.D.; Ribeiro, C. Commensal bacteria and essential amino acids control food choice behavior and reproduction. PLoS Biol. 2017, 15, e2000862. [CrossRef]

5. Turnbaugh, P.J.; Ley, R.E.; Mahowald, M.A.; Magrini, V.; Mardis, E.R.; Gordon, J.I. An obesity-associated gut microbiome with increased capacity for energy harvest. Nature 2006, 444, 1027-1131. [CrossRef]

6. Flint, H.J.; Duncan, S.H.; Louis, P. The impact of nutrition on intestinal bacterial communities. Curr. Opin. Microbiol. 2017, 38, 59-65. [CrossRef]

7. Ridaura, V.K.; Faith, J.J.; Rey, F.E.; Cheng, J.; Duncan, A.E.; Kau, A.L.; Griffin, N.W.; Lombard, V.; Henrissat, B.; Bain, J.R. Gut microbiota from twins discordant for obesity modulate metabolism in mice. Science 2013, 341, 1241214. [CrossRef]

8. Tilg, H.; Moschen, A.R. Gut Microbiome, Obesity, and Metabolic Syndrome. In Metabolic Syndrome: A Comprehensive Textbook; Ahima, R.S., Ed.; Springer: Cham, Switzerland, 2016; pp. 447-459.

9. Morais, P.B.; Martins, M.B.; Klaczko, L.B.; Mendonça-Hagler, L.C.; Hagler, A.N. Yeast succession in the Amazon fruit Parahancornia amapa as resource partitioning among Drosophila spp. Appl. Environ. Microbiol. 1995, 61, 4251-4257. [CrossRef]

10. Ryu, J.-H.; Kim, S.-H.; Lee, H.-Y.; Bai, J.Y.; Nam, Y.-D.; Bae, J.-W.; Lee, D.G.; Shin, S.C.; Ha, E.-M.; Lee, W.-J. Innate immune homeostasis by the homeobox gene caudal and commensal-gut mutualism in Drosophila. Science 2008, 319, 777-782. [CrossRef]

11. Ridley, E.V.; Wong, A.C.; Westmiller, S.; Douglas, A.E. Impact of the resident microbiota on the nutritional phenotype of Drosophila melanogaster. PLoS ONE 2012, 7, e36765. [CrossRef]

12. Wong, A.C.-N.; Luo, Y.; Jing, X.; Franzenburg, S.; Bost, A.; Douglas, A.E. The host as the driver of the microbiota in the gut and external environment of Drosophila melanogaster. Appl. Environ. Microbiol. 2015, 81, 6232-6240. [CrossRef] [PubMed]

13. Newell, P.D.; Douglas, A.E. Interspecies interactions determine the impact of the gut microbiota on nutrient allocation in Drosophila melanogaster. Appl. Environ. Microbiol. 2014, 80, 788-796. [CrossRef] [PubMed]

14. Dobson, A.J.; Chaston, J.M.; Newell, P.D.; Donahue, L.; Hermann, S.L.; Sannino, D.R.; Westmiller, S.; Wong, A.C.-N.; Clark, A.G.; Lazzaro, B.P. Host genetic determinants of microbiota-dependent nutrition revealed by genome-wide analysis of Drosophila melanogaster. Nat. Commun. 2015, 6, 6312. [CrossRef] [PubMed]

15. Huang, J.-H.; Douglas, A.E. Consumption of dietary sugar by gut bacteria determines Drosophila lipid content. Biol. Lett. 2015, 11, 20150469. [CrossRef]

16. Reed, L.K.; Lee, K.; Zhang, Z.; Rashid, L.; Poe, A.; Hsieh, B.; Deighton, N.; Glassbrook, N.; Bodmer, R.; Gibson, G. Systems genomics of metabolic phenotypes in wild-type Drosophila melanogaster. Genetics 2014, 197, 781-793. [CrossRef]

17. Birse, R.T.; Choi, J.; Reardon, K.; Rodriguez, J.; Graham, S.; Diop, S.; Ocorr, K.; Bodmer, R.; Oldham, S. High-fat-diet-induced obesity and heart dysfunction are regulated by the TOR pathway in Drosophila. Cell Metab. 2010, 12, 533-544. [CrossRef]

18. Dew-Budd, K.; Jarnigan, J.; Reed, L.K. Genetic and sex-specific transgenerational effects of a high fat diet in Drosophila melanogaster. PLoS ONE 2016, 11, e0160857. [CrossRef]

19. Wong, A.C.-N.; Dobson, A.J.; Douglas, A.E. Gut microbiota dictates the metabolic response of Drosophila to diet. J. Exp. Biol. 2014, 217, 1894-1901. [CrossRef]

20. Chaston, J.M.; Newell, P.D.; Douglas, A.E. Metagenome-wide association of microbial determinants of host phenotype in Drosophila melanogaster. MBio 2014, 5, e01631-14. [CrossRef]

21. Chaston, J.M.; Dobson, A.J.; Newell, P.D.; Douglas, A.E. Host genetic control of the microbiota mediates the Drosophila nutritional phenotype. Appl. Environ. Microbiol. 2016, 82, 671-679. [CrossRef]

22. Early, A.M.; Shanmugarajah, N.; Buchon, N.; Clark, A.G. Drosophila Genotype Influences Commensal Bacterial Levels. PLoS ONE 2017, 12, e0170332. [CrossRef] [PubMed]

23. Shin, S.C.; Kim, S.-H.; You, H.; Kim, B.; Kim, A.C.; Lee, K.-A.; Yoon, J.-H.; Ryu, J.-H.; Lee, W.-J. Drosophila microbiome modulates host developmental and metabolic homeostasis via insulin signaling. Science 2011, 334, 670-674. [CrossRef]

24. Chandler, J.A.; Lang, J.M.; Bhatnagar, S.; Eisen, J.A.; Kopp, A. Bacterial communities of diverse Drosophila species: Ecological context of a host-microbe model system. PLoS Genet. 2011, 7, e1002272. [CrossRef] [PubMed] 
25. Vacchini, V.; Gonella, E.; Crotti, E.; Prosdocimi, E.M.; Mazzetto, F.; Chouaia, B.; Callegari, M.; Mapelli, F.; Mandrioli, M.; Alma, A. Bacterial diversity shift determined by different diets in the gut of the spotted wing fly Drosophila suzukii is primarily reflected on acetic acid bacteria. Environ. Microbiol. Rep. 2017, 9, 91-103. [CrossRef] [PubMed]

26. Tefit, M.; Gillet, B.; Joncour, P.; Hughes, S.; Leulier, F. Stable association of a Drosophila-derived microbiota with its animal partner and the nutritional environment upon transfer between populations and generations. bioRxiv 2017, arXiv:111492.

27. Mendez, S.; Watanabe, L.; Hill, R.; Owens, M.; Moraczewski, J.; Rowe, G.C.; Riddle, N.C.; Reed, L.K. The TreadWheel: A novel apparatus to measure genetic variation in response to gently induced exercise for Drosophila. PLoS ONE 2016, 11, e0164706. [CrossRef]

28. Carvalho, G.B.; Kapahi, P.; Benzer, S. Compensatory ingestion upon dietary restriction in Drosophila melanogaster. Nat. Methods 2005, 2, 813. [CrossRef]

29. Leboffe, M.J.; Pierce, B.E. Microbiology: Laboratory Theory and Application; Morton Publishing Company: Englewood, CO, USA, 2012.

30. Maturin, L.; Peeler, J.T. BAM Aerobic Plate Count; US Food and Drug Administration: Silver Spring, MD, USA, 2001.

31. Mackay, T.F.; Richards, S.; Stone, E.A.; Barbadilla, A.; Ayroles, J.F.; Zhu, D.; Casillas, S.; Han, Y.; Magwire, M.M.; Cridland, J.M. The Drosophila melanogaster genetic reference panel. Nature 2012, 482, 173. [CrossRef]

32. Huang, W.; Massouras, A.; Inoue, Y.; Peiffer, J.; Ràmia, M.; Tarone, A.M.; Turlapati, L.; Zichner, T.; Zhu, D.; Lyman, R.F. Natural variation in genome architecture among 205 Drosophila melanogaster Genetic Reference Panel lines. Genome Res. 2014, 24, 1193-1208. [CrossRef]

33. Ashburner, M. Drosophila. A laboratory Handbook; Cold Spring Harbor Laboratory Press: Cold Spring, Harbor, NY, USA, 1989.

34. Clark, A.G.; Keith, L.E. Variation among extracted lines of Drosophila melanogaster in triacylglycerol and carbohydrate storage. Genetics 1988, 119, 595-607.

35. De Luca, M.; Yi, N.; Allison, D.B.; Leips, J.; Ruden, D.M. Mapping quantitative trait loci affecting variation in Drosophila triacylglycerol storage. Obes. Res. 2005, 13, 1596-1605. [CrossRef] [PubMed]

36. Reed, L.K.; Williams, S.; Springston, M.; Brown, J.; Freeman, K.; DesRoches, C.E.; Sokolowski, M.B.; Gibson, G. Genotype-by-diet interactions drive metabolic phenotype variation in Drosophila melanogaster. Genetics 2010, 185, 1009-1019. [CrossRef] [PubMed]

37. Bradford, M.M. A rapid and sensitive method for the quantitation of microgram quantities of protein utilizing the principle of protein-dye binding. Anal. Biochem. 1976, 72, 248-254. [CrossRef]

38. Rulifson, E.J.; Kim, S.K.; Nusse, R.J.S. Ablation of insulin-producing neurons in flies: Growth and diabetic phenotypes. Science 2002, 296, 1118-1120. [CrossRef]

39. Kumar, R.; Eipers, P.; Little, R.B.; Crowley, M.; Crossman, D.K.; Lefkowitz, E.J.; Morrow, C.D. Getting started with microbiome analysis: Sample acquisition to bioinformatics. Curr. Protoc. Hum. Genet. 2014, 82, 18.8.1-18.8.29. [CrossRef]

40. Bolger, A.M.; Lohse, M.; Usadel, B.J.B. Trimmomatic: A flexible trimmer for Illumina sequence data. Bioinformatics 2014, 30, 2114-2120. [CrossRef]

41. Caporaso, J.G.; Kuczynski, J.; Stombaugh, J.; Bittinger, K.; Bushman, F.D.; Costello, E.K.; Fierer, N.; Pena, A.G.; Goodrich, J.K.; Gordon, J.I. QIIME allows analysis of high-throughput community sequencing data. Nat. Methods 2010, 7, 335. [CrossRef]

42. Edgar, R.C.J.B. Search and clustering orders of magnitude faster than BLAST. Bioinformatics 2010, 26, $2460-2461$. [CrossRef]

43. Quast, C.; Pruesse, E.; Yilmaz, P.; Gerken, J.; Schweer, T.; Yarza, P.; Peplies, J.; Glöckner, F.O. The SILVA ribosomal RNA gene database project: Improved data processing and web-based tools. Nucleic Acids Res. 2012, 41, D590-D596. [CrossRef]

44. Caporaso, J.G.; Bittinger, K.; Bushman, F.D.; DeSantis, T.Z.; Andersen, G.L.; Knight, R.J.B. PyNAST: A flexible tool for aligning sequences to a template alignment. Bioinformatics 2010, 26, 266-267. [CrossRef]

45. Price, M.N.; Dehal, P.S.; Arkin, A.P. FastTree: Computing large minimum evolution trees with profiles instead of a distance matrix. Mol. Biol. Evol. 2009, 26, 1641-1650. [CrossRef] 
46. Bruno, D.; Bonelli, M.; De Filippis, F.; Di Lelio, I.; Tettamanti, G.; Casartelli, M.; Ercolini, D.; Caccia, S. The intestinal microbiota of Hermetia illucens larvae is affected by diet and shows a diverse composition in the different midgut regions. Appl. Environ. Microbiol. 2019, 85, e01864-18. [CrossRef]

47. Wu, G.D.; Chen, J.; Hoffmann, C.; Bittinger, K.; Chen, Y.-Y.; Keilbaugh, S.A.; Bewtra, M.; Knights, D.; Walters, W.A.; Knight, R.J.S. Linking long-term dietary patterns with gut microbial enterotypes. Science 2011, 334, 105-108. [CrossRef]

48. Bruce-Keller, A.J.; Salbaum, J.M.; Luo, M.; Blanchard, E., IV; Taylor, C.M.; Welsh, D.A.; Berthoud, H.-R. Obese-type gut microbiota induce neurobehavioral changes in the absence of obesity. Biol. Psychiatry 2015, 77, 607-615. [CrossRef] [PubMed]

49. Klepsatel, P.; Procházka, E.; Gáliková, M. Crowding of Drosophila larvae affects lifespan and other life-history traits via reduced availability of dietary yeast. Exp. Gerontol. 2018, 110, 298-308. [CrossRef] [PubMed]

50. Bing, X.; Gerlach, J.; Loeb, G.; Buchon, N. Nutrient-dependent impact of microbes on Drosophila suzukii development. MBio 2018, 9, e02199-17. [CrossRef] [PubMed]

51. Skorupa, D.A.; Dervisefendic, A.; Zwiener, J.; Pletcher, S.D. Dietary composition specifies consumption, obesity, and lifespan in Drosophila melanogaster. Aging Cell 2008, 7, 478-490. [CrossRef]

52. Sang, J.H. The quantitative nutritional requirements of Drosophila melanogaster. J. Exp. Biol. 1956, 33, 45-72.

53. Sgro, C.M.; Partridge, L. Evolutionary responses of the life history of wild-caught Drosophila melanogaster to two standard methods of laboratory culture. Am. Nat. 2000, 156, 341-353. [CrossRef]

54. Hoffmann, A.A.; Hallas, R.; Sinclair, C.; Partridge, L. Rapid loss of stress resistance in Drosophila melanogaster under adaptation to laboratory culture. Evolution 2001, 55, 436-438. [CrossRef]

55. Russell, T.; Kurtz, R. A Comparison of Laboratory-Reared Stock and Captured Fruit Flies (Drosophila melanogaster) using Upward Movement, Phototaxic, and Starvation Assays Reveals Significant Behavioral Differences. Staff Rev. 2012, 6. Available online: https://www.commackschools.org/Downloads/Fruit $\% 20 F l y \%$ 20Trinity\%20Russell.pdf (accessed on 4 October 2020).

56. Staubach, F.; Baines, J.F.; Künzel, S.; Bik, E.M.; Petrov, D.A. Host species and environmental effects on bacterial communities associated with Drosophila in the laboratory and in the natural environment. PLoS ONE 2013, 8, e70749. [CrossRef] [PubMed]

57. Pais, I.S.; Valente, R.S.; Sporniak, M.; Teixeira, L. Drosophila melanogaster establishes a species-specific mutualistic interaction with stable gut-colonizing bacteria. PLoS Biol. 2018, 16, e2005710. [CrossRef] [PubMed]

58. Urbaniec, K.; Zalewski, P.; Zhu, X.X. A decomposition approach for retrofit design of energy systems in the sugar industry. Appl. Therm. Eng. 2000, 20, 1431-1442. [CrossRef]

59. Hillier, R.M.; Lyster, R.L.J. Whey protein denaturation in heated milk and cheese whey. J. Dairy Res. 1979, 46, 95-102. [CrossRef]

60. Adair, K.L.; Wilson, M.; Bost, A.; Douglas, A.E. Microbial community assembly in wild populations of the fruit fly Drosophila melanogaster. ISME J. 2018, 1, 959-972. [CrossRef]

61. Douglas, A.E. The Drosophila model for microbiome research. Lab Anim. 2018, 47, 157-164. [CrossRef]

62. Wong, A.C.; Chaston, J.M.; Douglas, A.E. The inconstant gut microbiota of Drosophila species revealed by 16S rRNA gene analysis. ISME J. 2013, 7, 1922. [CrossRef]

63. Corby-Harris, V.; Pontaroli, A.C.; Shimkets, L.J.; Bennetzen, J.L.; Habel, K.E.; Promislow, D.E. Geographical distribution and diversity of bacteria associated with natural populations of Drosophila melanogaster. Appl. Environ. Microbiol. 2007, 73, 3470-3479. [CrossRef]

64. Jehrke, L.; Stewart, F.A.; Droste, A.; Beller, M. The impact of genome variation and diet on the metabolic phenotype and microbiome composition of Drosophila melanogaster. Sci. Rep. 2018, 8, 1-15. [CrossRef]

65. Wong, C.N.A.; Ng, P.; Douglas, A.E. Low-diversity bacterial community in the gut of the fruitfly Drosophila melanogaster. Environ. Microbiol. 2011, 13, 1889-1900. [CrossRef] [PubMed]

66. Moghadam, N.N.; Thorshauge, P.M.; Kristensen, T.N.; de Jonge, N.; Bahrndorff, S.; Kjeldal, H.; Nielsen, J.L. Strong responses of Drosophila melanogaster microbiota to developmental temperature. Fly 2018, 12, 1-12. [CrossRef] [PubMed]

67. Martinez-Porchas, M.; Villalpando-Canchola, E.; Suarez, L.E.O.; Vargas-Albores, F. How conserved are the conserved 16S-rRNA regions? Peer] 2017, 5, e3036. [CrossRef] [PubMed]

68. Behar, A.; Jurkevitch, E.; Yuval, B. Bringing back the fruit into fruit fly-bacteria interactions. Mol. Ecol. 2008, 17, 1375-1386. [CrossRef] 
69. Ferguson, L.V.; Dhakal, P.; Lebenzon, J.E.; Heinrichs, D.E.; Bucking, C.; Sinclair, B.J.J.F.E. Seasonal shifts in the insect gut microbiome are concurrent with changes in cold tolerance and immunity. Funct. Ecol. 2018, 32, 2357-2368. [CrossRef]

70. Tong, Q.; Zhang, J. Effects of captivity and season on the gut microbiota of the brown frog (Rana dybowskii). Front. Microbiol. 2019, 10, 1912. [CrossRef]

71. Maurice, C.F.; Knowles, S.C.; Ladau, J.; Pollard, K.S.; Fenton, A.; Pedersen, A.B.; Turnbaugh, P.J. Marked seasonal variation in the wild mouse gut microbiota. ISME J. 2015, 9, 2423-2434. [CrossRef]

72. Parks, B.W.; Nam, E.; Org, E.; Kostem, E.; Norheim, F.; Hui, S.T.; Pan, C.; Civelek, M.; Rau, C.D.; Bennett, B.J. Genetic control of obesity and gut microbiota composition in response to high-fat, high-sucrose diet in mice. Cell Metab. 2013, 17, 141-152. [CrossRef]

73. Zhang, C.; Zhang, M.; Wang, S.; Han, R.; Cao, Y.; Hua, W.; Mao, Y.; Zhang, X.; Pang, X.; Wei, C. Interactions between gut microbiota, host genetics and diet relevant to development of metabolic syndromes in mice. ISME J. 2010, 4, 232-241. [CrossRef]

74. Kreznar, J.H.; Keller, M.P.; Traeger, L.L.; Rabaglia, M.E.; Schueler, K.L.; Stapleton, D.S.; Zhao, W.; Vivas, E.I.; Yandell, B.S.; Broman, A.T. Host genotype and gut microbiome modulate insulin secretion and diet-induced metabolic phenotypes. Cell Rep. 2017, 18, 1739-1750. [CrossRef]

75. Rosenthal, N.; Brown, S. The mouse ascending: Perspectives for human-disease models. Nat. Cell Biol. 2007, 9, 993-999. [CrossRef] [PubMed]

76. Berger, Z.; Ttofi, E.K.; Michel, C.H.; Pasco, M.Y.; Tenant, S.; Rubinsztein, D.C.; O’Kane, C.J. Lithium rescues toxicity of aggregate-prone proteins in Drosophila by perturbing Wnt pathway. Hum. Mol. Genet. 2005, 14, 3003-3011. [CrossRef] [PubMed]

77. Martin, T.L.; Balser, S.R.; Young, G.S.; Lewis, S.D. Cost and effectiveness of commercially available nesting substrates for Deer Mice (Peromyscus maniculatus). J. Am. Assoc. Lab. Anim. Sci. 2016, 55, 412-418. [PubMed]

78. King, E.G.; Macdonald, S.J.; Long, A.D. Properties and power of the Drosophila Synthetic Population Resource for the routine dissection of complex traits. Genetics 2012, 191, 935-949. [CrossRef] [PubMed]

79. Williams, S.; Dew-Budd, K.; Davis, K.; Anderson, J.; Bishop, R.; Freeman, K.; Davis, D.; Bray, K.; Perkins, L.; Hubickey, J. Metabolomic and gene expression profiles exhibit modular genetic and dietary structure linking metabolic syndrome phenotypes in Drosophila. G3 Genes Genomes Genet. 2015, 5, 2817-2829. [CrossRef]

80. Martyn, J.J.; Kaneki, M.; Yasuhara, S.; Warner, D.S.; Warner, M.A. Obesity-induced insulin resistance and hyperglycemia: Etiological factors and molecular mechanisms. J. Am. Soc. Anesthesiol. 2008, 109, 137-148. [CrossRef]

81. Akter, R.; Nessa, A.; Husain, M.; Wahed, F.; Khatun, N.; Yesmin, M.; Nasreen, S.; Tajkia, T. Effect of Obesity on Fasting Blood Sugar. Mymensingh Med. J. 2017, 26, 7-11.

82. Million, M.; Maraninchi, M.; Henry, M.; Armougom, F.; Richet, H.; Carrieri, P.; Valero, R.; Raccah, D.; Vialettes, B.; Raoult, D. Obesity-associated gut microbiota is enriched in Lactobacillus reuteri and depleted in Bifidobacterium animalis and Methanobrevibacter smithii. Int. J. Obes. 2012, 36, 817-825. [CrossRef]

83. Ignacio, A.; Fernandes, M.R.; Rodrigues, V.A.; Groppo, F.C.; Cardoso, A.L.; Avila-Campos, M.; Nakano, V. Correlation between body mass index and faecal microbiota from children. Clin. Microbiol. Infect. 2016, 22, 258.e1-258.e8. [CrossRef]

84. Murugesan, S.; Ulloa-Martínez, M.; Martínez-Rojano, H.; Galván-Rodríguez, F.; Miranda-Brito, C.; Romano, M.; Piña-Escobedo, A.; Pizano-Zárate, M.; Hoyo-Vadillo, C.; García-Mena, J. Study of the diversity and short-chain fatty acids production by the bacterial community in overweight and obese Mexican children. Eur. J. Clin. Microbiol. Infect. Dis. 2015, 34, 1337-1346. [CrossRef]

85. Armougom, F.; Henry, M.; Vialettes, B.; Raccah, D.; Raoult, D. Monitoring bacterial community of human gut microbiota reveals an increase in Lactobacillus in obese patients and Methanogens in anorexic patients. PLoS ONE 2009, 4, e7125. [CrossRef]

86. Schwiertz, A.; Taras, D.; Schäfer, K.; Beijer, S.; Bos, N.A.; Donus, C.; Hardt, P.D. Microbiota and SCFA in lean and overweight healthy subjects. Obesity 2010, 18, 190-195. [CrossRef] [PubMed]

87. Ley, R.E.; Turnbaugh, P.J.; Klein, S.; Gordon, J.I. Human gut microbes associated with obesity. Nature 2006, 444, 1022-1023. [CrossRef] [PubMed] 
88. Furet, J.-P.; Kong, L.-C.; Tap, J.; Poitou, C.; Basdevant, A.; Bouillot, J.-L.; Mariat, D.; Corthier, G.; Doré, J.; Henegar, C. Differential adaptation of human gut microbiota to bariatric surgery-induced weight loss. Diabetes 2010, 59, 3049-3057. [CrossRef] [PubMed]

89. Storelli, G.; Defaye, A.; Erkosar, B.; Hols, P.; Royet, J.; Leulier, F.J.C.m. Lactobacillus plantarum promotes Drosophila systemic growth by modulating hormonal signals through TOR-dependent nutrient sensing. Cell Metab. 2011, 14, 403-414. [CrossRef] [PubMed]

90. Pawitan, Y.; Michiels, S.; Koscielny, S.; Gusnanto, A.; Ploner, A. False discovery rate, sensitivity and sample size for microarray studies. Bioinformatics 2005, 21, 3017-3024. [CrossRef] [PubMed]

Publisher's Note: MDPI stays neutral with regard to jurisdictional claims in published maps and institutional affiliations.

(C) 2020 by the authors. Licensee MDPI, Basel, Switzerland. This article is an open access article distributed under the terms and conditions of the Creative Commons Attribution (CC BY) license (http://creativecommons.org/licenses/by/4.0/). 